\title{
CELLLIKE MAPPINGS AND NONMETRIZABLE COMPACTA OF FINITE COHOMOLOGICAL DIMENSION
}

\author{
SIBE MARDEŠIĆ AND LEONARD R. RUBIN
}

\begin{abstract}
Compact Hausdorff spaces $X$ of cohomological dimension $\operatorname{dim}_{\mathbf{Z}} X$ $\leq n$ are characterized as cell-like images of compact Hausdorff spaces $Z$ with covering dimension $\operatorname{dim} Z \leq n$. The proof essentially uses the newly developed techniques of approximate inverse systems.
\end{abstract}

\section{INTRODUCTION}

In 1978, R. D. Edwards [2] announced the result that every metrizable compact space $X$ of cohomological dimension $\operatorname{dim}_{\mathrm{Z}} X \leq n$ (integer coefficients) is the image of a cell-like mapping $f: Z \rightarrow X$ of a compact metric space $Z$ with $\operatorname{dim} Z \leq n$. A proof of this result was published in 1981 by J. J. Walsh [16]. By the classical Vietoris-Begle theorem (see, e.g. [15, Chapter 6, $§ 9$, Theorem 15]), the converse also holds, and one thus has a characterization of metrizable compacta $X$ with $\operatorname{dim}_{\mathrm{Z}} X \leq n$.

More recently, L. R. Rubin and P. J. Schapiro [14] have succeeded in generalizing the Edwards-Walsh theorem to the case of metrizable spaces $X$ and $Z$.

The purpose of our present paper is to generalize the Edwards-Walsh theorem in another direction, i.e., to establish the result for compact Hausdorff spaces (see $\S 11$, Theorem 3).

In generalizing the theorem to compact Hausdorff spaces, one encounters a difficulty which was not present in the two previous cases. In the case of metric compacta [16], the space $X$ was represented as the limit of an inverse sequence $\mathbf{X}$ of polyhedra. This sequence led to another sequence $\mathbf{Z}$ of polyhedra of dimension $\leq n$, and the space $Z$ was obtained as the limit of $\mathbf{Z}$.

Dealing with a noncompact situation, Rubin and Schapiro [14] had to overcome many obstacles. Still they were able to obtain $Z$ as the inverse limit

Received by the editors October 5, 1988.

1980 Mathematics Subject Classification (1985 Revision). Primary 54F45, 55M10, 54B25.

Key words and phrases. Cell-like map, cohomological dimension, covering dimension, approximate inverse system, inverse system, compact Hausdorff space.

This paper is the result of the cooperation of the authors during the period February-October 1987, while S. Mardešic (on leave from the University of Zagreb) was visiting the University of Oklahoma in Norman and the University of Washington in Seattle. L. Rubin acknowledges support in the form of a travel grant from the University of Oklahoma Research Council. 
of a sequence $\mathbf{Z}$ of noncompact polyhedra because the uniform structure of a metric space has a countable basis of uniform coverings.

In the case of compact Hausdorff spaces $X$, one cannot avoid using partially ordered inverse systems $\mathbf{X}$ of polyhedra. Upon applying the process of approximating the bonding maps of $\mathbf{X}$ on $n$-skeleta as in [16,14], one invariably obtains a system $\mathbf{Z}$ whose bonding maps $r_{a_{1} a_{2}}$ do not satisfy the commutativity condition $r_{a_{1} a_{2}} r_{a_{2} a_{3}}=r_{a_{1} a_{3}}, a_{1} \leq a_{2} \leq a_{3}$. The best one can achieve is to keep the distances $d\left(r_{a_{1} a_{2}} r_{a_{2} a_{3}}, r_{a_{1} a_{3}}\right)$ small. Therefore, the attempt to prove the theorem for compact Hausdorff spaces had to be preceded by the development of a theory of approximately commutative inverse systems. This was initiated by the authors in [6], and it was continued in [8, 9, 10].

An interesting phenomenon, which adds additional difficulty, is that the approximate system yielding $Z$, constructed herein, is a system of metric compacta $Z_{a}^{*}($ see $\S 6)$ which are not polyhedra.

Recently, A. N. Dranishnikov [1] solved a classical problem of P. S. Aleksandrov by exhibiting a metric compactum $X$ with $\operatorname{dim}_{\mathrm{z}} X \leq 3$ and $\operatorname{dim} X=\infty$. This shows that $\operatorname{dim}_{z}$ and dim differ and in our main result (Theorem 3 ) one cannot take for $f$ the identity map $1_{X}$.

\section{APPROXIMATE INVERSE SYSTEMS}

We quote from [6] the basic definition of an approximate system and of its limit.

Definition 1. An approximate (inverse) system of metric compacta $\mathbf{X}=$ $\left(X_{a}, \varepsilon_{a}, p_{a a^{\prime}}, A\right)$ consists of the following: A directed ordered set $(A, \leq)$ with no maximal element; for each $a \in A$, a compact metric space $X_{a}$ with metric $d=d_{a}$ and a real number $\varepsilon_{a}>0$; for each pair $a \leq a^{\prime}$ from $A$, a mapping $p_{a a^{\prime}}: X_{a^{\prime}} \rightarrow X_{a}$, satisfying the following conditions:

(A1) $d\left(p_{a_{1} a_{2}} p_{a_{2} a_{3}}, p_{a_{1} a_{3}}\right) \leq \varepsilon_{a_{1}}, a_{1} \leq a_{2} \leq a_{3} ; p_{a a}=\mathrm{id}$.

(A2) $(\forall a \in A)(\forall \eta>0)\left(\exists a^{\prime} \geq a\right)\left(\forall a_{2} \geq a_{1} \geq a^{\prime}\right)$

$$
d\left(p_{a a_{1}} p_{a_{1} a_{2}}, p_{a a_{2}}\right) \leq \eta \text {. }
$$

$$
\begin{aligned}
& (\forall a \in A)(\forall \eta>0)\left(\exists a^{\prime} \geq a\right)\left(\forall a^{\prime \prime} \geq a^{\prime}\right)\left(\forall x, x^{\prime} \in X_{a^{\prime \prime}}\right) \\
& d\left(x, x^{\prime}\right) \leq \varepsilon_{a^{\prime \prime}} \Rightarrow d\left(p_{a a^{\prime \prime}}(x), p_{a a^{\prime \prime}}\left(x^{\prime}\right)\right) \leq \eta .
\end{aligned}
$$

We refer to the numbers $\varepsilon_{a}$ as the meshes of $\mathbf{X}$. We say $\mathbf{X}$ is cofinite if $A$ is cofinite, i.e., every element $a \in A$ has only finitely many predecessors.

If $\pi_{a}: \prod_{a \in A} X_{a} \rightarrow X_{a}, a \in A$, denote projections, we define the limit space $X=\lim \mathbf{X}$ and the natural projections $p_{a}: X \rightarrow X_{a}$ as follows.

Definition 2. A point $x=\left(x_{a}\right) \in \prod X_{a}$ belongs to $X=\lim \mathbf{X}$ provided for every $a \in A$,

$$
x_{a}=\lim _{a_{1}} p_{a a_{1}}\left(x_{a_{1}}\right) .
$$

The natural projection $p_{a}=\pi_{a} \mid X: X \rightarrow X_{a}$. 
We now quote (as propositions) several results from [6 and 8] needed in this paper.

Proposition 1. If $\mathbf{X}=\left(X_{a}, \varepsilon_{a}, p_{a a^{\prime}}, A\right)$ is an approximate system and $X_{a} \neq \phi$ for each $a \in A$, then $X=\lim \mathbf{X}$ is a compact Hausdorff space and $X \neq \phi$ (see [6, Theorems 1 and 2]).

Proposition 2. If $\mathscr{V}_{a}$ is a basis for $X_{a}, a \in A$, then the sets $p_{a}^{-1}\left(V_{a}\right), V_{a} \in \mathscr{V}_{a}$, $a \in A$, form a basis for $X=\lim \mathbf{X}$ (see [6, Lemma 3]).

Proposition 3. For any $a \in A, \lim _{a_{1}} d\left(p_{a}, p_{a a_{1}} p_{a_{1}}\right)=0$, where $d(f, g)=$ $\sup \{d(f(x), g(x)): x \in X\} \quad($ see $[6$, Lemma 4$])$.

Proposition 4. Every approximate system $\mathbf{X}$ has the following two properties:

(B1) Let $a \in A$ and let $U \subseteq X_{a}$ be an open set which contains $p_{a}(X)$. Then there exists an $a^{\prime} \geq a$ such that $p_{a a^{\prime \prime}}\left(X_{a^{\prime \prime}}\right) \subseteq U$ for any $a^{\prime \prime} \geq a^{\prime}$.

(B2) For every open covering $\mathscr{U}$ of $X$, there exists an $a \in A$ such that for any $a_{1} \geq a$ there exists an open covering $\mathscr{V}$ of $X_{a_{1}}$ for which $\left(p_{a_{1}}\right)^{-1}(\mathscr{V})$ refines $\mathscr{U}$ (see [8, Theorem 3] and [6, Theorem 1]).

Proposition 5. Let $\mathbf{X}=\left(X_{a}, \varepsilon_{a}, p_{a a^{\prime}}, A\right)$ be an approximate system with limit $X$. If $\operatorname{dim} X_{a} \leq n$ for all $a \in A$, then the covering dimension $\operatorname{dim} X \leq n$ (see [6, Theorem 4]).

Proposition 6. Let $\mathbf{X}=\left(X_{a}, \varepsilon_{a}, p_{a a^{\prime}}, A\right)$ be an approximate system with limit $X$, and let $B \subseteq A$ be a cofinal subset of $A$. Then $\mathbf{Y}=\left(X_{b}, \varepsilon_{b}, p_{b b^{\prime}}, B\right)$ is also an approximate system. Moreover, the restriction $p=\pi \mid X$ of the projection $\pi: \prod_{a \in A} X_{a} \rightarrow \prod_{b \in B} X_{b}$ is a homeomorphism $p: X \rightarrow Y$ (see [8, Proposition 2]).

We will now add several new propositions.

Proposition 7. Every approximate system $\mathbf{X}$ has the following property:

(R1) For every $\varepsilon>0$, every compact ANR $P$, and every mapping $h: X \rightarrow P$, there is an $a \in A$ such that for any $a^{\prime} \geq a$ there is a mapping $f: X_{a^{\prime}} \rightarrow$ $P$ which satisfies $d\left(f p_{a^{\prime}}, h\right) \leq 2 \varepsilon$.

Proof. (This proof follows closely that given for the analogous theorem for commutative systems given in [7, Chapter I, §5.2, Theorem 8]. We first embed $P$ in the Hilbert cube $Q$ and choose a closed neighborhood $G$ of $P$ in $Q$ which admits a retraction $r: G \rightarrow P$. Then we choose $\delta>0$ so small that $\delta \leq \varepsilon / 2$, the $\delta$-neighborhood of $P$ is contained in $G$, and

$$
y, y^{\prime} \in G, \quad d\left(y, y^{\prime}\right) \leq \delta \Rightarrow d\left(r(y), r\left(y^{\prime}\right)\right) \leq \varepsilon / 2 .
$$

We then choose an open covering $\mathscr{U}$ of $X$ so fine that each $h(U), U \in \mathscr{U}$, is contained in a convex set $B \subseteq G$ with $\operatorname{diam} B<\delta$.

By property (B2) (Proposition 4) there is an $a_{0} \in A$ such that there is an open covering $\mathscr{W}$ of $X_{a_{0}}$ for which $\left(p_{a_{0}}\right)^{-1}(\mathscr{W})$ refines $\mathscr{U}$. Let $\mathscr{W}_{1}$ be a finite 
open covering of $X_{a_{0}}$ such that $\mathscr{W}_{1}$ is a star-refinement of $\mathscr{W}$. Let $\mathscr{W}_{2} \subseteq \mathscr{W}_{1}$ consist of all $W \in \mathscr{W}_{1}$ with $W \cap p_{a_{0}}(X) \neq \phi$. For each $W \in \mathscr{W}_{2}$ we choose a point $y_{w} \in h\left(\left(p_{a_{0}}\right)^{-1}(W)\right)$. Let $N$ be a closed neighborhood of $p_{a_{0}}(X)$ in $X_{a_{0}}$ covered by $\mathscr{W}_{2}$, and let $\left(\varphi_{W}, W \in \mathscr{W}_{2}\right)$ be a partition of unity on $N$ subordinated to the cover $\mathscr{W}_{2} \mid N$. We define a map $g: N \rightarrow Q \subseteq \mathbf{R}^{\infty}$ by

$$
g(z)=\sum_{W \in \mathscr{W}_{2}} \varphi_{w}(z) y_{w}, \quad z \in N
$$

It is not difficult to show that

$$
g(N) \subseteq G
$$

Moreover,

$$
d\left(r g p_{a_{0}}, h\right) \leq \varepsilon
$$

(see [7, pp. 63-64]).

We now apply property (B1) (Proposition 4) and find an $a \geq a_{0}$ such that for any $a^{\prime} \geq a$ one has

$$
p_{a_{0} a^{\prime}}\left(X_{a^{\prime}}\right) \subseteq N
$$

Using Proposition 3, we can also assume that

$$
d\left(p_{a_{0}}, p_{a_{0} a^{\prime}} p_{a^{\prime}}\right) \leq \omega,
$$

where $\omega>0$ is such that

$$
z, z^{\prime} \in N, \quad d\left(z, z^{\prime}\right) \leq \omega \Rightarrow d\left(r g(z), r g\left(z^{\prime}\right)\right) \leq \varepsilon .
$$

Then, (6) and (7) yield

$$
d\left(r g p_{a_{0}}, r g p_{a_{0} a^{\prime}} p_{a^{\prime}}\right) \leq \varepsilon .
$$

Putting

$$
f=r g p_{a_{0} a^{\prime}}: X_{a^{\prime}} \rightarrow P
$$

one obtains

$$
d\left(f p_{a^{\prime}}, h\right) \leq 2 \varepsilon, \quad a^{\prime} \geq a
$$

Proposition 8. Let $\mathbf{X}=\left(X_{a}, \varepsilon_{a}, p_{a a^{\prime}}, A\right)$ be an approximate system of metric compacta with the following property:

$$
\begin{gathered}
\left(\forall a_{1}\right)\left(\exists a_{1}^{\prime} \geq a_{1}\right)\left(\forall a_{2} \geq a_{1}^{\prime}\right)\left(\exists a_{2}^{\prime} \geq a_{2}\right)\left(\forall a_{3} \geq a_{2}^{\prime}\right) \\
p_{a_{1} a_{2}} p_{a_{2} a_{3}} \simeq 0 .
\end{gathered}
$$

Then $X=\lim \mathbf{X}$ has the shape of a point, $\operatorname{sh}(X)=0$.

Proof. Let $P$ be an ANR and $f: X \rightarrow P$ be a map. It suffices to prove that $f \simeq 0$. Choose $\delta>0$ such that $\delta$-near maps into $P$ are homotopic. By 
property (R1) (Proposition 7), there is an $a_{1} \in A$ and a map $g: X_{a_{1}} \rightarrow P$ such that $d\left(g p_{a_{1}}, f\right) \leq \delta$ and therefore $g p_{a_{1}} \simeq f$. It therefore suffices to prove that

$$
g p_{a_{1}} \simeq 0 \text {. }
$$

Let $\eta>0$ be such that

$$
d\left(x, x^{\prime}\right) \leq 2 \eta \Rightarrow d\left(g(x), g\left(x^{\prime}\right)\right) \leq \delta .
$$

Then for any map $p_{a_{1}}^{\prime}: X \rightarrow X_{a_{1}}$,

$$
d\left(p_{a_{1}}^{\prime}, p_{a_{1}}\right) \leq 2 \eta \Rightarrow d\left(g p_{a_{1}}^{\prime}, g p_{a_{1}}\right) \leq \delta
$$

and therefore,

$$
d\left(p_{a_{1}}^{\prime}, p_{a_{1}}\right) \leq 2 \eta \Rightarrow g p_{a_{1}}^{\prime} \simeq g p_{a_{1}} .
$$

We now choose an $a_{1}^{\prime} \geq a_{1}$ according to the assumption of the proposition. Next we choose $a_{2} \geq a_{1}^{\prime}$ in such a way that

$$
d\left(p_{a_{1} a_{3}} p_{a_{3} a_{4}}, p_{a_{1} a_{4}}\right) \leq \eta,
$$

for all $a_{4} \geq a_{3} \geq a_{2}$ (property (A2)). Clearly, for any $a_{3} \geq a_{2}$, (15) implies

$$
d\left(p_{a_{1} a_{2}} p_{a_{2} a_{3}}, p_{a_{1} a_{3}}\right) \leq \eta \text {. }
$$

Moreover, (15) and Proposition 3 imply

$$
d\left(p_{a_{1} a_{3}} p_{a_{3}}, p_{a_{1}}\right) \leq \eta \text {. }
$$

(16) and (17) yield

$$
d\left(p_{a_{1} a_{2}} p_{a_{2} a_{3}} p_{a_{3}}, p_{a_{1}}\right) \leq 2 \eta
$$

which, by (14), implies

$$
g p_{a_{1}} \simeq g p_{a_{1} a_{2}} p_{a_{2} a_{3}} p_{a_{3}}, \quad \text { for any } a_{3} \geq a_{2} .
$$

We now choose an $a_{2}^{\prime} \geq a_{2}$ according to the assumption of the proposition. Therefore, if we choose $a_{3} \geq a_{2}^{\prime}$, we have

$$
p_{a_{1} a_{2}} p_{a_{2} a_{3}} \simeq 0 \text {. }
$$

Now, (11) follows from (19) and (20).

Remark 1. An analogous proposition holds for the following property and any $n \geq 1$ :

$$
\begin{gathered}
\left(\forall a_{1}\right)\left(\exists a_{1}^{\prime} \geq a_{1}\right) \cdots\left(\forall a_{n} \geq a_{n-1}^{\prime}\right)\left(\exists a_{n}^{\prime} \geq a_{n}\right)\left(\forall a_{n+1} \geq a_{n}^{\prime}\right) \\
p_{a_{1} a_{2}} p_{a_{2} a_{3}} \ldots p_{a_{n}} p_{a_{n+1}} \simeq 0 .
\end{gathered}
$$

Proposition 9. Let $\mathbf{X}=\left(X_{a}, \varepsilon_{a}, p_{a a^{\prime}}, A\right)$ be an approximate system with limit $X$ and projections $p_{a}$. Let $<^{\prime}$ be a binary relation on $A$ satisfying the following conditions:
(i) $a_{1}<a_{2} \Rightarrow a_{1}<a_{2}$,
(ii) $a_{1}<{ }^{\prime} a_{2}$ and $a_{2} \leq a_{3} \Rightarrow a_{1}<^{\prime} a_{3}$,
(iii) $(\forall a \in A)\left(\exists a^{\prime} \in A\right) a<^{\prime} a^{\prime}$. 
Write $a_{1} \leq a_{2}$ if $a_{1}<^{\prime} a_{2}$ or $a_{1}=a_{2}$, and let $A^{\prime}$ be the set $A$ provided with the relation $\leq^{\prime}$. Then $A^{\prime}$ is a directed set with no maximal element and $\mathbf{X}^{\prime}=\left(X_{a}, \varepsilon_{a}, p_{a a^{\prime}}, A^{\prime}\right)$ is an approximate system with limit $X^{\prime}$ and projections $p_{a}^{\prime}$. Moreover, $X^{\prime}=X$ and $p_{a}^{\prime}=p_{a}$.

Proof. If $a_{1}<^{\prime} a_{2}$ and $a_{2}<^{\prime} a_{3}$, then by (i), $a_{2}<a_{3}$ and by (ii), $a_{1}<^{\prime} a_{3}$. Therefore $\leq^{\prime}$ is transitive. For any $a_{1}, a_{2} \in A$, by (iii) there exist indexes $a_{1}^{\prime}, a_{2}^{\prime} \in A$ such that $a_{1}<^{\prime} a_{1}^{\prime}, a_{2}<^{\prime} a_{2}^{\prime}$. Since $(A, \leq)$ is directed, there is an $a^{\prime \prime} \in A$ such that $a_{1}^{\prime} \leq a^{\prime \prime}, a_{2}^{\prime} \leq a^{\prime \prime}$. Now (ii) implies $a_{1}<a^{\prime \prime}, a_{2}<^{\prime} a^{\prime \prime}$, which proves that $A^{\prime}$ is directed.

We now verify that $\mathbf{X}^{\prime}$ is an approximate system. (A1) is an immediate consequence of (i). For given $a \in A$ and $\eta>0$, choose $a^{\prime} \geq a$ in accordance with (A2) for $\mathbf{X}$. By directedness of $A^{\prime}$, there is an $a_{1}^{\prime} \in A$ such that $a \leq^{\prime} a_{1}^{\prime}$ and $a^{\prime} \leq^{\prime} a_{1}^{\prime}$. If $a_{1}^{\prime} \leq^{\prime} a_{1} \leq^{\prime} a_{2}$, then $a^{\prime} \leq a_{1} \leq a_{2}$ and therefore

$$
d\left(p_{a a_{1}} p_{a_{1} a_{2}}, p_{a a_{2}}\right) \leq \eta,
$$

as required by (A2) for $\mathbf{X}^{\prime}$.

If $a^{\prime} \geq a$ satisfies (A3) for $\mathbf{X}$, then we choose $a_{1}^{\prime}$ so that $a \leq^{\prime} a_{1}^{\prime}$ and $a^{\prime} \leq a_{1}^{\prime}$. For any $a^{\prime \prime}$ with $a_{1}^{\prime} \leq^{\prime} a^{\prime \prime}$ we have $a_{1}^{\prime} \leq a^{\prime \prime}$ and therefore,

$$
d\left(x, x^{\prime}\right) \leq \varepsilon_{a^{\prime \prime}} \Rightarrow d\left(p_{a a^{\prime \prime}}(x), p_{a a^{\prime \prime}}\left(x^{\prime}\right)\right) \leq \eta,
$$

as required by (A3) for $\mathbf{X}^{\prime}$.

Finally, for any $a \in A$,

$$
A_{a}^{\prime}=\left\{a_{1} \in A: a \leq^{\prime} a_{1}\right\} \subseteq A_{a}=\left\{a_{1} \in A: a \leq a_{1}\right\}
$$

and the set $A_{a}^{\prime}$ is cofinal in $A_{a}$. Therefore,

$$
\lim _{a_{1} \in A_{a}^{\prime}} p_{a a_{1}}\left(x_{a_{1}}\right)=\lim _{a_{1} \in A_{a}} p_{a a_{1}}\left(x_{a_{1}}\right) \quad \text { for any }\left(x_{a}\right) \in \prod X_{a} .
$$

By Definition 2, this shows that $X=X^{\prime}$ and thus also $p_{a}=p_{a}^{\prime}$.

Proposition 10. Let $\mathbf{X}=\left(X_{a}, \varepsilon_{a}, p_{a a^{\prime}}, A\right)$ be an approximate system of metric compacta $X_{a}$ with metrics $d_{a}$. Then there exist metrics $d_{a}^{\prime} \leq 1$ on $X_{a}$, defining the same topology on $X_{a}$, and there exist numbers $\varepsilon_{a}^{\prime}>0$ such that $\mathbf{X}^{\prime}=$ $\left(X_{a}, \varepsilon_{a}^{\prime}, p_{a a^{\prime}}, A\right)$ is also an approximate system. Moreover, $X^{\prime}=X$ and $p_{a}^{\prime}=$ $p_{a}, a \in A$.

Proof. For each $a \in A$ we put

$$
\begin{aligned}
d_{a}^{\prime} & =\frac{d_{a}}{1+d_{a}}, \\
\varepsilon_{a}^{\prime} & =\frac{\varepsilon_{a}}{1+\varepsilon_{a}} .
\end{aligned}
$$


Note that $d_{a}^{\prime} \leq 1$ and $d_{a}^{\prime}$ is a metric compatible with $d_{a}$. Moreover,

$$
\begin{gathered}
d_{a}^{\prime} \leq d_{a}, \\
d_{a}\left(x, x^{\prime}\right) \leq \varepsilon_{a} \Leftrightarrow d_{a}^{\prime}\left(x, x^{\prime}\right) \leq \varepsilon_{a}^{\prime} .
\end{gathered}
$$

The verification of (A1)-(A3) for $\mathbf{X}^{\prime}$ is now straightforward. Moreover, $X^{\prime}=$ $X, p_{a}^{\prime}=p_{a}$, because the limit space depends only on the topology of the spaces $X_{a}$ and on the maps $p_{a a^{\prime}}$.

\section{REPRESENTING COMPACT SPACES AS APPROXIMATE LIMITS}

It is well known that every compact Hausdorff space $X$ is the limit of a (commutative) inverse system of compact polyhedra $\mathbf{X}=\left(X_{a}, p_{a a^{\prime}}, A\right)$ (with PL bonding maps $p_{a a^{\prime}}$ ) (see, e.g., [7, I, §5.2, Theorem 7]). However, B. A. Pasynkov has shown $[11,12]$ that there exist compact Hausdorff spaces $X$ which are not obtainable as limits of inverse systems $\mathbf{X}$ of polyhedra with surjective bonding mappings $p_{a a^{\prime}}$. This difficulty vanishes if one considers approximate inverse systems as we show in the following theorem (needed later).

Theorem 1. Every compact Hausdorff space $X$ is the limit of an approximate (cofinite) inverse system $\mathbf{X}=\left(X_{a}, \varepsilon_{a}, p_{a a^{\prime}}, A\right)$, where the spaces $X_{a}$ are polyhedra and all the bonding maps $p_{a a^{\prime}}$ are (irreducible) surjective PL-maps. Moreover, the cardinal card $(A) \leq w(X)$, the weight of $X$.

We recall some notions and simple facts needed in the proof. By a polyhedron we always mean a compact polyhedron and by a complex, a finite simplicial complex. If $K$ is a complex, then $|K|$ denotes its carrier, i.e., the corresponding polyhedron.

Let $K$ be a complex and let $f, g: X \rightarrow|K|$ be mappings. We say that $g$ is a $K$-modification of $f$ if for every point $x \in X$ and simplex $\sigma \in K, f(x) \in \sigma$ implies $g(x) \in \sigma$. Note that a simplicial approximation $\varphi: K_{1} \rightarrow K_{2}$ of a mapping $\pi:\left|K_{1}\right| \rightarrow\left|K_{2}\right|$ is a $K_{2}$-modification of $\pi$. Moreover, if $K^{\prime}$ is a subdivision of $K$ and $g: X \rightarrow\left|K^{\prime}\right|$ is a $K^{\prime}$-modification of $f: X \rightarrow\left|K^{\prime}\right|=|K|$, then $g$ is also a $K$-modification of $f$.

We say that a mapping $f: X \rightarrow|K|$ is $K$-irreducible if for every $K$-modification $g$ of $f$ one has $g(X)=|K|$. Since $f$ is its own $K$-modification, a $K$ irreducible map $f$ is onto. A mapping $f: X \rightarrow P$ into a polyhedron is called irreducible if it is $K$-irreducible for some triangulation $K$ of $P$. Note that every irreducible map $f: X \rightarrow P$ is onto.

For every complex $K$ and mapping $f: X \rightarrow|K|$ there is a subcomplex $L \subseteq K$ and a $K$-modification $g: X \rightarrow|L| \subseteq|K|$ which is $L$-irreducible and, therefore $g: X \rightarrow|L|$ is irreducible and onto. If $f$ is already $K$-irreducible, we put $L=K, g=f$. If not, there is a $K$-modification $f_{1}$ of $f$ with $f_{1}(X) \neq|K|$. Clearly $f_{1}(X)$ can be "pushed off" some principal simplex $\sigma \in K$ ( $\sigma$ is not a proper face of some $\tau \in K$ ). $f_{1}$ is a $K$-modification of $f$ and the 
carrier of $f_{1}(X)$ (i.e., the minimal subcomplex of $K$ containing $f_{1}(X)$ ) has fewer simplexes than the carrier of $f(X)$. After finitely many steps the process stops and we obtain the desired subcomplex $L$ and the desired map $g$.

If $f: X \rightarrow|K|$ is $K$-irreducible and $K^{\prime}$ is a subdivision of $K$, then $f$ is also $K^{\prime}$-irreducible. Indeed, every $K^{\prime}$-modification $g: X \rightarrow\left|K^{\prime}\right|$ of $f$ is also a $K$-modification and, therefore, $g(X)=|K|=\left|K^{\prime}\right|$.

Our proof of Theorem 1 is based on the following lemma.

Lemma 1. Let $X$ be a compact Hausdorff space, let $f_{i}: X \rightarrow P_{i}$ be maps to polyhedra $P_{i}$ and $\varepsilon_{i}>0, i=1, \ldots, k$. Then there exist a polyhedron $Q$, an irreducible (onto) map $g: X \rightarrow Q$ and PL-mappings $p_{i}: Q \rightarrow P_{i}$ such that $d\left(f_{i}, p_{i} g\right) \leq \varepsilon_{i}, i=1, \ldots, k$. Moreover, if for a given index $i$ the mapping $f_{i}$ is irreducible, then the corresponding mapping $p_{i}$ is also irreducible and therefore onto.

Proof of Lemma 1. For each $i \in\{1, \ldots, k\}$ choose a triangulation $K_{i}$ of $P_{i}$. If $f_{i}$ is irreducible, let $f_{i}$ be $K_{i}$-irreducible. Let $L_{i}$ be a subdivision of $K_{i}$ with

$$
\operatorname{mesh} L_{i} \leq \varepsilon_{i} / 2 \text {. }
$$

Note that $f_{i}$ is $L_{i}$-irreducible if it is irreducible.

Let $P=P_{1} \times \cdots \times P_{k}$, let $f=f_{1} \times \cdots \times f_{k}: X \rightarrow P$ and let $\pi_{i}: P \rightarrow P_{i}, i=$ $1, \ldots, k$, be the projections. Choose $\delta>0$ so small that

$$
d\left(x, x^{\prime}\right) \leq \delta \Rightarrow d\left(\pi_{i}(x), \pi_{i}\left(x^{\prime}\right)\right) \leq \varepsilon_{i} / 2, \quad i=1, \ldots, k .
$$

Let $K$ be a triangulation of $P$ so fine that

$$
\operatorname{mesh} K \leq \delta \text {, }
$$

and the projections $\pi_{i}:|K| \rightarrow\left|L_{i}\right|$ admit simplicial approximations $p_{i}: K \rightarrow$ $L_{i}, i=1, \ldots, k$. Since $p_{i}$ is an $L_{i}$-modification of $\pi_{i}$, we have

$$
d\left(p_{i}, \pi_{i}\right) \leq \operatorname{mesh} L_{i} \leq \varepsilon_{i} / 2 .
$$

There exists a subcomplex $L \subseteq K$ and a $K$-modification $g: X \rightarrow|L|$ of $f$ such that $g$ is $L$-irreducible. Putting $Q=|L|$, we see that $g: X \rightarrow Q$ is irreducible (and onto). Note that $d(f, g) \leq \operatorname{mesh} K \leq \delta$, and therefore,

$$
d\left(\pi_{i} f, \pi_{i} g\right) \leq \varepsilon_{i} / 2, \quad i=1, \ldots, k .
$$

Since $\pi_{i} f=f_{i},(4)$ and (5) yield

$$
d\left(f_{i}, p_{i} g\right) \leq \varepsilon_{i}, \quad i=1, \ldots, k .
$$

We will now show that $p_{i} g$ is an $L_{i}$-modification of $\pi_{i} f=f_{i}$. Let $x \in X$ and let $\sigma \in K$ be the carrier of $f(x)$. Let $\sigma_{i}=p_{i}(\sigma) \in L_{i}$. Then $\sigma_{i}$ is the carrier of $p_{i} f(x)$. Since $p_{i}$ is an $L_{i}$-modification of $\pi_{i}$, we conclude that $\sigma_{i}$ is a face of the carrier $\tau_{i} \in L_{i}$ of $\pi_{i} f(x)=f_{i}(x)$. Since $g$ is a $K$-modification of $f$, we have $g(x) \in \sigma$ and therefore $p_{i} g(x) \in p_{i}(\sigma)=\sigma_{i} \leq \tau_{i}$. This shows 
that $p_{i} g(x)$ belongs to the carrier $\tau_{i}$ of $f_{i}(x)$ in $\left|L_{i}\right|$ and therefore $p_{i} g$ is indeed an $L_{i}$-modification of $f_{i}$.

We will now show that $p_{i}:|L| \rightarrow\left|L_{i}\right|$ is $L_{i}$-irreducible if $f_{i}: X \rightarrow P_{i}$ is irreducible. In this case we already know that $f_{i}$ is $L_{i}$-irreducible. Moreover, for any $L_{i}$-modification $q_{i}:|L| \rightarrow\left|L_{i}\right|$ of $p_{i}$, the mapping $q_{i} g$ is an $L_{i}$ modification of $p_{i} g$ and therefore also an $L_{i}$-modification of $f_{i}$. This then implies $q_{i}(|L|)=q_{i} g(X)=\left|L_{i}\right|$.

Proof of Theorem 1. Repeat (with obvious modifications) the proof of Theorem 5 of [6] or the proof of Theorem 3 of [8]. Use Lemma 1 instead of Lemma 5 (in the first case) and Lemma 2 (in the second case). Note that in Theorem 5 of [6] the bonding maps are not required to be onto. In Theorem 3 of [8] the bonding maps are onto but need not be PL-maps. Moreover, this result does not apply to the class of all polyhedra.

\section{COHOMOLOGICAL DIMENSION OF LIMITS OF APPROXIMATE SYSTEMS}

For compact Hausdorff spaces $X$, one can define the cohomological dimension $\operatorname{dim}_{\mathrm{z}} X$ (integer coefficients) by putting $\operatorname{dim}_{\mathrm{z}} X \leq n, n \geq 1$, provided every map $f: A \rightarrow K(\mathbf{Z}, n)$ from a closed subset $A$ of $X$ to an Eilenberg-Mac Lane complex $K(\mathbf{Z}, n)$ admits an extension $\tilde{f}: X \rightarrow K(\mathbf{Z}, n)$ (see, e.g., [4, Remark 5 and Theorem 26] or [3]). In [5], $\operatorname{dim}_{\mathrm{z}} X \leq n$ was characterized by an approximate factorization property, which we will now describe.

Definition 3. A map $p: Q \rightarrow P$ between polyhedra is called $(n, \varepsilon)$-approximable, $\varepsilon>0, n \geq 1$, provided for every triangulation $M$ of $Q$ there is a PL-mapping $p^{\prime}:\left|M^{(n+1)}\right| \rightarrow P$ of the $(n+1)$-skeleton of $M$ such that

$$
\begin{gathered}
d\left(p^{\prime}, p|| M^{(n+1)} \mid\right) \leq \varepsilon, \\
\operatorname{dim} p^{\prime}\left(\left|M^{(n+1)}\right|\right) \leq n .
\end{gathered}
$$

The following proposition was proved in [5] as Theorem 1.

Proposition 11. A compact Hausdorff space $X$ has cohomological dimension $\operatorname{dim}_{\mathrm{z}} X \leq n, n \geq 1$, if and only if for every polyhedron $P$, every map $f: X \rightarrow$ $P$, and every $\varepsilon>0$, there is a polyhedron $Q$ and there are maps $g: X \rightarrow$ $Q, p: Q \rightarrow P$ such that

$$
d(p g, f) \leq \varepsilon,
$$

and $p$ is $(n, \varepsilon)$-approximable.

Using Proposition 11 we will now give a criterion for determining whether $\operatorname{dim}_{\mathrm{z}} X \leq n$, when $X$ is the limit of an approximate system of polyhedra.

Theorem 2. Let $\mathbf{X}=\left(X_{a}, \varepsilon_{a}, p_{a a^{\prime}}, A\right)$ be an approximate system of polyhedra. The limit $X=\lim \mathbf{X}$ satisfies $\operatorname{dim}_{\mathbf{z}} X \leq n, n \geq 1$, if and only if for every $a \in A$ 
and every $\eta>0$, there is an $a^{\prime} \geq a$ such that for every $a^{\prime \prime} \geq a^{\prime}$ the mapping $p_{a a^{\prime \prime}}$ is $(n, \eta)$-approximable.

Proof of necessity. Let $\operatorname{dim}_{\mathrm{z}} X \leq n$ and let $a \in A, \eta>0$ be given. By (A2) there is an $a_{1} \geq a$ such that for any $a^{\prime} \geq a_{1}$ one has

$$
d\left(p_{a a^{\prime}} p_{a^{\prime} a^{\prime \prime}}, p_{a a^{\prime \prime}}\right) \leq \eta / 7, \quad a^{\prime \prime} \geq a^{\prime} \geq a_{1} .
$$

Note that (4) implies

$$
d\left(p_{a a^{\prime}} p_{a^{\prime} a^{\prime \prime}} p_{a^{\prime \prime}}, p_{a a^{\prime \prime}} p_{a^{\prime \prime}}\right) \leq \eta / 7, \quad a^{\prime \prime} \geq a^{\prime} .
$$

Passing to the limit with $a^{\prime \prime}$ and taking into account Proposition 3, one obtains

$$
d\left(p_{a a^{\prime}} p_{a^{\prime}}, p_{a}\right) \leq \eta / 7, \quad a^{\prime} \geq a_{1} .
$$

By Proposition 11, there is a polyhedron $Q$ and there are maps $g: X \rightarrow$ $Q, p: Q \rightarrow X_{a}$ such that

$$
d\left(p_{a}, p g\right) \leq \eta / 7
$$

and $p$ is $(n, \eta / 7)$-approximable.

Choose $\delta>0$ so small that

$$
d\left(x, x^{\prime}\right) \leq \delta \Rightarrow d\left(p(x), p\left(x^{\prime}\right)\right) \leq \eta / 7 .
$$

By Property ( $\mathrm{R} 1$ ) (Proposition 7), there is an $a^{\prime} \geq a_{1}$ such that there is a mapping $p^{\prime}: X_{a^{\prime}} \rightarrow Q$ satisfying

$$
d\left(p^{\prime} p_{a^{\prime}}, g\right) \leq \delta .
$$

Now (8) and (9) imply

$$
d\left(p p^{\prime} p_{a^{\prime}}, p g\right) \leq \eta / 7 \text {. }
$$

Note that (10), (7) and (6) yield

$$
d\left(p p^{\prime} p_{a^{\prime}}, p_{a a^{\prime}} p_{a^{\prime}}\right) \leq 3 \eta / 7 .
$$

By (11) there is a neighborhood $U$ of $p_{a^{\prime}}(X)$ in $X_{a^{\prime}}$ such that

$$
d\left(p p^{\prime}\left|U, p_{a a^{\prime}}\right| U\right) \leq 4 \eta / 7 \text {. }
$$

By Property (B1) (Proposition 4), there is an $a_{1}^{\prime} \geq a^{\prime}$ such that for any $a^{\prime \prime} \geq a_{1}^{\prime}$ one has

$$
p_{a^{\prime} a^{\prime \prime}}\left(X_{a^{\prime \prime}}\right) \subseteq U
$$

and therefore,

$$
d\left(p p^{\prime} p_{a^{\prime} a^{\prime \prime}}, p_{a a^{\prime}} p_{a^{\prime} a^{\prime \prime}}\right) \leq 4 \eta / 7 .
$$

Note that (14) and (4) yield

$$
d\left(p p^{\prime} p_{a^{\prime} a^{\prime \prime}}, p_{a a^{\prime \prime}}\right) \leq 5 \eta / 7 .
$$


We will show that $p_{a a^{\prime \prime}}$ is $(n, \eta)$-approximable for any $a^{\prime \prime} \geq a_{1}^{\prime}$.

Let $M$ be any triangulation of $X_{a^{\prime \prime}}$. Choose a triangulation $N$ of $Q$ so fine that $\operatorname{mesh}(N) \leq \delta$. Let $M^{\prime}$ be a subdivision of $M$ so fine that $p^{\prime} p_{a^{\prime} a^{\prime \prime}}: X_{a^{\prime \prime}} \rightarrow$ $Q$ admits a simplicial approximation $q: M^{\prime} \rightarrow N$. Note that the $(n+1)$ skeleton $\left|M^{(n+1)}\right| \subseteq\left|M^{\prime(n+1)}\right|$ and

$$
q\left(\left|M^{(n+1)}\right|\right) \subseteq q\left(\left|M^{(n+1)}\right|\right) \subseteq\left|N^{(n+1)}\right| .
$$

Moreover,

$$
d\left(q, p^{\prime} p_{a^{\prime} a^{\prime \prime}}\right) \leq \operatorname{mesh}(N) \leq \delta .
$$

Therefore (8) implies

$$
d\left(p q, p p^{\prime} p_{a^{\prime} a^{\prime \prime}}\right) \leq \eta / 7
$$

(15) and (18) imply

$$
d\left(p q, p_{a a^{\prime \prime}}\right) \leq 6 \eta / 7
$$

Since $p$ is $(n, \eta / 7)$-approximable, there is a PL-mapping $p^{*}:\left|N^{(n+1)}\right| \rightarrow X_{a}$ such that

$$
\begin{gathered}
d\left(p^{*}, p|| N^{(n+1)} \mid\right) \leq \eta / 7 \\
\operatorname{dim} p^{*}\left(\left|N^{(n+1)}\right|\right) \leq n
\end{gathered}
$$

By (16), $p^{*} q|| M^{(n+1)} \mid$ is a well-defined PL-mapping $\left|M^{(n+1)}\right| \rightarrow X_{a}$. Formulas (19) and (20) imply

$$
d\left(p_{a a^{\prime \prime}}|| M^{(n+1)}\left|, p^{*} q\right|\left|M^{(n+1)}\right|\right) \leq \eta .
$$

Moreover, (16) and (21) imply

$$
\operatorname{dim} p^{*} q\left(\left|M^{(n+1)}\right|\right) \leq n .
$$

This shows that $p^{*} q|| M^{(n+1)} \mid$ is an $(n, \eta)$-approximation of $p_{a a^{\prime \prime}}$.

Proof of sufficiency. Let $f: X \rightarrow P$ be a mapping into a polyhedron $P$ and let $\eta>0$. By Proposition 11 it suffices to exhibit an $a^{\prime \prime} \in A$ and a mapping $p: X_{a^{\prime \prime}} \rightarrow P$ such that

$$
d\left(p p_{a^{\prime \prime}}, f\right) \leq \eta
$$

and $p$ is $(n, \eta)$-approximable.

By Property (R1) (Proposition 7), there is a mapping $g: X_{a} \rightarrow P$ such that

$$
d\left(f, g p_{a}\right) \leq \eta / 2 .
$$

By simplicial approximation we can achieve that $g$ is a PL-mapping. Let $\delta>0$ be such that

$$
d\left(x, x^{\prime}\right) \leq \delta \Rightarrow d\left(g(x), g\left(x^{\prime}\right)\right) \leq \eta / 2
$$


By Proposition 3, there is an $a^{\prime} \geq a$ such that for any $a^{\prime \prime} \geq a^{\prime}$ one has

$$
d\left(p_{a a^{\prime \prime}} p_{a^{\prime \prime}}, p_{a}\right) \leq \delta
$$

and therefore,

$$
d\left(g p_{a a^{\prime \prime}} p_{a^{\prime \prime}}, g p_{a}\right) \leq \eta / 2 .
$$

By assumption there is an $a^{\prime \prime} \geq a^{\prime}$ for which $p_{a a^{\prime \prime}}$ is $(n, \delta)$-approximable. If we put $p=g p_{a a^{\prime \prime}}: X_{a^{\prime \prime}} \rightarrow P,(28)$ and (25) imply (24). It remains to show that $p$ is $(n, \eta)$-approximable.

Let $M$ be a triangulation of $X_{a^{\prime \prime}}$. Since $p_{a a^{\prime \prime}}$ is $(n, \delta)$-approximable, there is a PL-mapping $p^{\prime}:\left|M^{(n+1)}\right| \rightarrow X_{a}$ such that

$$
\begin{gathered}
d\left(p^{\prime}, p_{a a^{\prime \prime}}|| M^{(n+1)} \mid\right) \leq \delta, \\
\operatorname{dim} p^{\prime}\left(\left|M^{(n+1)}\right|\right) \leq n .
\end{gathered}
$$

Note that $g p^{\prime}:\left|M^{(n+1)}\right| \rightarrow P$ is a PL-map. (29) and (26) imply

$$
d\left(g p^{\prime}, p|| M^{(n+1)} \mid\right) \leq \eta \text {. }
$$

Moreover, (30) and the fact that $g$ is a PL-map imply

$$
\operatorname{dim} g p^{\prime}\left(\left|M^{(n+1)}\right|\right) \leq n .
$$

(31) and (32) prove that $p$ is indeed $(n, \eta)$-approximable.

Remark 2. Theorem 2 is a generalization of R. D. Edwards' criterion for the limit $X$ of an inverse sequence of polyhedra to satisfy $\operatorname{dim}_{\mathrm{z}} X \leq n$ (see [16, Theorem 4.2]).

\section{THE $n$-DIMENSIONAL CORE OF A COMPLEX}

In this section we will associate with every complex $K$ and every integer $n \geq 0$ a compact metric space $Z_{K}=Z_{K}^{(n)}$ with $\operatorname{dim} Z_{K} \leq n$, called the $n$ dimensional core of $K$.

Let $K, K^{\prime}, K^{2}, \ldots, K^{k}, \ldots$ denote the iterated barycentric subdivisions of $K$. For each $k \geq 0$ choose a simplicial approximation $q_{k k+1}: K^{k+1} \rightarrow K^{k}$ of the identity map $1:\left|K^{k+1}\right| \rightarrow\left|K^{k}\right|$ and let $q_{k k+j}=q_{k+1} \ldots q_{k+j-1 k+j}: K^{k+j}$ $\rightarrow K^{k}$. Note that $q_{k k+j}$ is a simplicial approximation of the identity $1:\left|K^{k+j}\right|$ $\rightarrow\left|K^{k}\right|$ and is therefore a $K^{k}$-modification of 1 . Consequently,

$$
d\left(q_{k k+j}, 1\right) \leq \operatorname{mesh}\left(K^{k}\right), \quad j \geq 0 .
$$

Since the maps $q_{k+1}$ are simplicial we have

$$
q_{k k+1}\left(\left(K^{k+1}\right)^{(n)}\right) \subseteq\left(K^{k}\right)^{(n)},
$$

where $L^{(n)}$ denotes the $n$-skeleton of $L$. Therefore we have an inverse sequence of polyhedra

$$
\mathbf{K}=\left(\left|\left(K^{k}\right)^{(n)}\right|, q_{k k+1}\right) .
$$


The $n$-dimensional core of $K$ is defined as the inverse limit

$$
Z_{K}=\lim \mathbf{K} \text {. }
$$

Since $\operatorname{dim}\left|\left(K^{k}\right)^{(n)}\right| \leq n$, we have

$$
\operatorname{dim} Z_{K} \leq n .
$$

We denote the natural projections from $Z_{K}$ to $\left|\left(K^{k}\right)^{(n)}\right|$ by $q_{k}$. Since the maps $q_{k k+1}$ are onto (Sperner's lemma, see, e.g., [15, Chapter 3, Ex. D3]), so are the maps $q_{k k+j}$ and $q_{k}$.

We now define a mapping $f_{K}: Z_{K} \rightarrow|K|$ by putting

$$
f_{K}=\lim _{k} q_{k} \text {. }
$$

Since $q_{k k+j} q_{k+j}=q_{k}$, (1) implies

$$
d\left(q_{k}, q_{k+j}\right) \leq \operatorname{mesh}\left(K^{k}\right), \quad j \geq 0,
$$

and since $\lim _{k} \operatorname{mesh}\left(K^{k}\right)=0$, we see that $\left(q_{k}\right)$ is a Cauchy sequence of maps $Z_{K} \rightarrow|K|$. Therefore $f_{K}$ exists and is continuous. Moreover, (7) implies

$$
d\left(q_{k}, f_{K}\right) \leq \operatorname{mesh}\left(K^{k}\right) .
$$

Since $q_{k}$ is onto and $\operatorname{mesh}\left(K^{k}\right) \rightarrow 0$, we see that $f_{K}\left(Z_{K}\right)$ is dense in $|K|$ and therefore $f_{K}: Z_{K} \rightarrow|K|$ is also an onto mapping.

Remark 3. The sequence $\left(\left|K^{k}\right|, \operatorname{mesh}\left(K^{k}\right)\right.$, id, N) is actually an approximate inverse sequence. Since $\mathbf{K}$ is a commutative cofinite sequence, one can provide it with meshes $\varepsilon_{k}$ and view $\mathbf{K}$ also as an approximate sequence. (7) shows that the inclusion maps $\left|\left(K^{k}\right)^{(n)}\right| \rightarrow\left|K^{k}\right|$ define a map of approximate systems. The existence of $f_{K}$ and its properties now follow from the general theory of maps between approximate systems (see [10 or 17]).

In our constructions in $\S 7$ we need to associate with every complex $K$ an $n$-dimensional metric compactum $Z_{K}^{*}$ which is a compactification of the topological sum of the $n$-skeleta $\left|\left(K^{k}\right)^{(n)}\right|$ of all the barycentric subdivisions $K^{k}$ of $K$, with remainder $Z_{K}$. We call $Z_{K}^{*}$ the stacked $n$-dimensional core of $K$.

$$
Z_{K}^{*}=\left(\bigoplus_{k \geq 0}\left|\left(K^{k}\right)^{(n)}\right|\right) \cup Z_{K} .
$$

To describe precisely the topology of $Z_{K}^{*}$ we form a new inverse sequence $\mathbf{K}^{*}=\left(\left|K^{* k}\right|, q_{k+1}^{*}\right)$, where

$$
K^{* k}=K^{(n)} \oplus\left(K^{\prime}\right)^{(n)} \oplus \cdots \oplus\left(K^{k}\right)^{(n)} .
$$

Note that

$$
\left|K^{* k+1}\right|=\left|K^{* k}\right| \oplus\left|\left(K^{k+1}\right)^{(n)}\right| .
$$


The bonding maps $q_{k k+1}^{*}:\left|K^{* k+1}\right| \rightarrow\left|K^{* k}\right|$ are defined by

$$
\begin{gathered}
q_{k k+1}^{*}||\left(K^{k+1}\right)^{(n)} \mid=q_{k k+1}, \\
q_{k k+1}^{*}|| K^{* k} \mid=\mathrm{id} .
\end{gathered}
$$

Finally, we put

$$
Z_{K}^{*}=\lim \mathbf{K}^{*}
$$

and denote the natural projections by $q_{k}^{*}: Z_{K}^{*} \rightarrow\left|K^{* k}\right|$. We have

$$
\operatorname{dim} Z_{K}^{*} \leq n,
$$

because $\operatorname{dim}\left|K^{* k}\right| \leq n$. Moreover,

$$
\begin{array}{cc}
Z_{K} \subseteq Z_{K}^{*}, \quad\left|K^{* k}\right| \subseteq Z_{K}^{*}, & k \geq 0, \\
q_{k}^{*}||\left(K^{k+j}\right)^{(n)} \mid=q_{k k+j}, & j \geq 0, \\
q_{k}^{*} \mid Z_{K}=q_{k} . &
\end{array}
$$

We now extend the mapping $f_{K}: Z_{K} \rightarrow|K|$ to $f_{K}^{*}: Z_{K}^{*} \rightarrow|K|$ by

$$
f_{K}^{*}=\lim _{k} q_{k}^{*} \text {. }
$$

Note that (12), (13), (7) imply

$$
d\left(q_{k}^{*}, q_{k+j}^{*}\right) \leq \operatorname{mesh}\left(K^{k}\right), j \geq 0,
$$

so that $f_{K}^{*}$ exists. Moreover, from (17) and (18)

$$
\begin{gathered}
f_{K}^{*}||\left(K^{k}\right)^{(n)} \mid \text { is inclusion into }|K|, \quad k \geq 0, \\
f_{K}^{*} \mid Z_{K}=f_{K} .
\end{gathered}
$$

Note that

$$
f_{K}^{*}\left(Z_{K}^{*}\right)=f_{K}\left(Z_{K}\right)=|K| .
$$

Also note that (20) implies

$$
d\left(q_{k}^{*}, f_{K}^{*}\right) \leq \operatorname{mesh}\left(K^{k}\right) .
$$

In the applications of these constructions in $\S 7$ we will also need a metric on $Z_{K}^{*}$. If we have a metric $d$ on $|K|$ such that the diameter $\operatorname{diam}|K| \leq 1$, then we can choose metrics $d^{*}$ on $Z_{K}^{*}$ and $d^{k}$ on $\left|K^{* k}\right|$ such that $\operatorname{diam} Z_{K}^{*} \leq 1$, $\operatorname{diam}\left|K^{* k}\right| \leq 1$, and

$$
d^{k}\left(q_{k}^{*}(x), q_{k}^{*}\left(x^{\prime}\right)\right) \leq d^{*}\left(x, x^{\prime}\right), \quad x, x^{\prime} \in Z_{K}^{*}, k \geq 0 .
$$

Indeed, we first define a metric $d^{k}$ on $\left|K^{* k}\right|=\left|K^{(n)}\right| \oplus \cdots \oplus\left|\left(K^{k}\right)^{(n)}\right|$ by putting $d^{k}(x, y)=\frac{1}{2^{k}} d(x, y)$ if $x, y$ belong to the same summand $\left|\left(K^{i}\right)^{(n)}\right| \subseteq|K|$, and 
$d^{k}(x, y)=\frac{1}{2^{k}}$ otherwise. Clearly $d^{k} \leq \frac{1}{2^{k}}$. We now define a metric $d^{*}$ on $\prod_{k \geq 0}\left|K^{* k}\right|$. If $x=\left(x^{k}\right), x^{\prime}=\left(x^{\prime k}\right) \in \Pi\left|K^{* k}\right|$, we put

$$
d^{*}\left(x, x^{\prime}\right)=\sup _{k} d^{k}\left(x^{k}, x^{\prime k}\right) \leq 1 \text {. }
$$

Since $Z_{K}^{*} \subseteq \prod\left|K^{* k}\right|, d^{*}$ is also a metric on $Z_{K}^{*}$ and diam $Z_{K}^{*} \leq 1$. Moreover, $x^{k}=q_{k}^{*}(x), x^{\prime k}=q_{k}^{*}\left(x^{\prime}\right)$, and we see that (26) implies (25).

Note that $d^{0}$ coincides with the metric $d$ on $|K|$ and therefore (25) yields

$$
d\left(q_{0}^{*}(x), q_{0}^{*}\left(x^{\prime}\right)\right) \leq d^{*}\left(x, x^{\prime}\right), \quad x, x^{\prime} \in Z_{K}^{*}
$$

\section{CONSTRUCTION OF THE APPROXIMATE SYSTEM $\mathbf{Z}$}

The following is an easy consequence of results already established.

Proposition 12. Let $X$ be a compact Hausdorff space with $\operatorname{dim}_{\mathrm{Z}} X=n \geq 1$. Then there exists an approximate inverse system $\mathbf{X}=\left(X_{a}, \varepsilon_{a}, p_{a a^{\prime}}, A\right)$ with $\lim \mathbf{X}=X$ such that

(i) $X_{a}$ is a polyhedron with a metric $d=d_{a} \leq 1$,

(ii) $\operatorname{dim} X_{a} \geq n \geq 1$,

(iii) $p_{a a^{\prime}}: X_{a^{\prime}} \rightarrow X_{a}$ is a surjective PL-mapping,

(iv) $\operatorname{card} A \leq w(X)$.

Proof. Theorem 1 yields a system $\mathbf{X}$ with $\lim \mathbf{X}=X, \operatorname{card} A \leq w(X)$, where $X_{a}$ are polyhedra and $p_{a a^{\prime}}$ are PL-surjections. By Proposition 10, one can assume that $d_{a} \leq 1$.

There is an index $a_{1} \in A$ such that for every $a \geq a_{1}$ one has $\operatorname{dim} X_{a} \geq n$. If this were not the case, then the set $B \subseteq A$ of all indexes $b \in A$ with $\operatorname{dim} X_{b} \leq n-1$ would be cofinal in $A$. Then Propositions 6 and 5 would imply $\operatorname{dim} X \leq n-1$. Since $\operatorname{dim}_{\mathrm{z}} X \leq \operatorname{dim} X$ (see [4]), we would have a contradiction with the assumption $\operatorname{dim}_{\mathrm{z}} X=n$. If we now restrict $A$ to the set of all $a \geq a_{1}$, we obtain an approximate system which satisfies all conditions (i)-(iv) (use Proposition 6 again).

From now on we assume that we have chosen a system $\mathbf{X}$ as in Proposition 12. We will now define an approximate system $\mathrm{Z}=\left(Z_{a}^{*}, \varepsilon_{a}, r_{a a^{\prime}}, A^{\prime}\right)$.

We first choose a triangulation $K_{a}$ for $X_{a}, a \in A$, such that

$$
6 \operatorname{mesh}\left(K_{a}\right) \leq \varepsilon_{a}, \quad a \in A .
$$

We next define the directed set $A^{\prime}$. As a set $A^{\prime}$ equals $A$, but $A^{\prime}$ has a new ordering $\leq^{\prime}$. In order to define it, we consider for any $a_{1}<a_{2}$ and any integer $k \geq 0$ the following three conditions:

(2) $d\left(p_{a_{1} a^{\prime}} p_{a^{\prime} a^{\prime \prime}}, p_{a_{1} a^{\prime \prime}}\right) \leq \operatorname{mesh}\left(K_{a_{1}}^{k}\right)$, for $a^{\prime \prime} \geq a^{\prime} \geq a_{2}$,

(3) $d\left(x, x^{\prime}\right) \leq \varepsilon_{a^{\prime \prime}} \Rightarrow d\left(p_{a_{1} a^{\prime \prime}}(x), p_{a_{1} a^{\prime \prime}}\left(x^{\prime}\right)\right) \leq \operatorname{mesh}\left(K_{a_{1}}^{k}\right)$, for $a^{\prime \prime} \geq a_{2}$,

(4) $p_{a_{1} a^{\prime \prime}}: X_{a^{\prime \prime}} \rightarrow X_{a_{1}}$ is $\left(n, \operatorname{mesh}\left(K_{a_{1}}^{k}\right)\right)$-approximable, for $a^{\prime \prime} \geq a_{2}$. 
We put $a_{1}<a_{2}$ provided $a_{1}<a_{2}$ and conditions (2), (3), (4) hold for $k=0$.

Lemma 2. The binary relation $<^{\prime}$ on $A$ has properties (i)-(iii) from Proposition 9, and therefore $A^{\prime}$ is a directed set with no maximal element. Moreover, for any $a_{1} \in A$ and integer $k \geq 0$ there exists an $a_{2}>a_{1}$ such that (2), (3) and (4) hold.

Proof. (i) and (ii) are obviously true and (iii) follows from the last assertion for $k=0$. To verify the latter, put

$$
\eta=\operatorname{mesh}\left(K_{a_{1}}^{k}\right)>0
$$

and apply Theorem 2. We obtain an $a_{2}>a_{1}$ such that for any $a^{\prime \prime} \geq a_{2}$ the mapping $p_{a_{1} a^{\prime \prime}}$ satisfies (4). However, by (A2) and (A3), one can assume that $a_{2}$ also satisfies (2) and (3).

Lemma 3. If $a_{1}<a_{2}$, the set of all integers $k \geq 0$, which satisfy (3) is finite.

Proof. Assume that there is an infinite sequence $k_{1}<k_{2}<\cdots$ of integers satisfying (3). Then for any two points $x, x^{\prime} \in X_{a_{2}}$

$$
d\left(x, x^{\prime}\right) \leq \varepsilon_{a_{2}} \Rightarrow p_{a_{1} a_{2}}(x)=p_{a_{1} a_{2}}\left(x^{\prime}\right) .
$$

This is so because, by (3),

$$
d\left(p_{a_{1} a_{2}}(x), p_{a_{1} a_{2}}\left(x^{\prime}\right)\right) \leq \operatorname{mesh}\left(K_{a_{1}}^{k_{i}}\right), \quad i=1,2, \ldots,
$$

and $\operatorname{mesh}\left(K_{a_{1}}^{k_{i}}\right) \rightarrow 0$ as $i \rightarrow \infty$. Consequently, $p_{a_{1} a_{2}}$ maps every component of $X_{a_{2}}$ to a single point. Since $X_{a_{1}}=p_{a_{1} a_{2}}\left(X_{a_{2}}\right)$, it follows that $X_{a_{1}}$ is a finite set of points, which contradicts (ii) of Proposition 12.

Whenever $a_{1}<^{\prime} a_{2}$, by definition of $<^{\prime}$ and Lemma 3 , there is a maximal integer $k \geq 0$ such that (2),(3) and (4) hold. We denote it by $k\left(a_{1}, a_{2}\right)$.

Lemma 4. If $a_{1}<a_{2}$, then (2), (3) and (4) hold for $k=k\left(a_{1}, a_{2}\right)$ and also

$$
d\left(p_{a_{1} a^{\prime}} p_{a^{\prime}}, p_{a_{1}}\right) \leq \operatorname{mesh}\left(K_{a_{1}}^{k\left(a_{1}, a_{2}\right)}\right), \quad \text { for } a^{\prime} \geq a_{2} \text {. }
$$

If $a_{1}<a_{2}$ and $a_{2} \leq a_{3}$, then

$$
k\left(a_{1}, a_{2}\right) \leq k\left(a_{1}, a_{3}\right) .
$$

Furthermore, for $a_{1} \in A$ and any integer $k \geq 0$ there is an $a_{2} \in A$ such that $a_{1}<a_{2}$ and

$$
k \leq k\left(a_{1}, a_{2}\right) \text {. }
$$

Proof. (2), (3) and (4) hold for $k=k\left(a_{1}, a_{2}\right)$ by the very definition of $k\left(a_{1}, a_{2}\right)$. By (2), one has

$$
d\left(p_{a_{1} a^{\prime}} p_{a^{\prime} a^{\prime \prime}} p_{a^{\prime \prime}}, p_{a_{1} a^{\prime \prime}} p_{a^{\prime \prime}}\right) \leq \operatorname{mesh}\left(K_{a_{1}}^{k\left(a_{1}, a_{2}\right)}\right), \quad \text { for } a^{\prime \prime} \geq a^{\prime} \geq a_{2} .
$$

Passing to the limit with $a^{\prime \prime}$ (using Proposition 3), one obtains (7). 
$k=k\left(a_{1}, a_{2}\right)$ satisfies (2), (3) and (4) also for $a_{1}$ and $a_{3}$. Therefore, (8) follows from the maximality of $k\left(a_{1}, a_{3}\right)$. By the last assertion of Lemma 2, for any $k \geq 0$ there exists an $a_{2}>a_{1}$ such that (2), (3) and (4) hold. Clearly, $a_{1}<a_{2}$. Now (9) follows from the maximality of $k\left(a_{1}, a_{2}\right)$.

Lemma 5. Let $K$ and $L$ be complexes and let $p:|K| \rightarrow|L|$ be an $(n, \operatorname{mesh}(L))$ approximable mapping. Then there exists a PL-mapping $g:\left|K^{(n+1)}\right| \rightarrow\left|L^{(n)}\right|$ such that

$$
d\left(g, p|| K^{n+1} \mid\right) \leq 2 \operatorname{mesh}(L) .
$$

Proof. By assumption, there is a PL-mapping $p^{\prime}:\left|K^{(n+1)}\right| \rightarrow|L|$ such that

$$
\begin{gathered}
d\left(p^{\prime}, p|| K^{(n+1)} \mid\right) \leq \operatorname{mesh}(L), \\
\operatorname{dim}\left(p^{\prime}\left(\left|K^{(n+1)}\right|\right)\right) \leq n .
\end{gathered}
$$

Let $\varphi: p^{\prime}\left(\left|K^{(n+1)}\right|\right) \rightarrow|L|$ be a simplicial approximation to the inclusion of $p^{\prime}\left(\left|K^{(n+1)}\right|\right)$ into $|L|$ relative to $L$. Then, $g=\varphi p^{\prime}:\left|K^{(n+1)}\right| \rightarrow\left|L^{(n)}\right|$ is a PL-mapping and

$$
d\left(g, p^{\prime}\right) \leq \operatorname{mesh}(L)
$$

Now (13) and (15) yield (12).

For $a_{1}<a_{2}$, we define a PL-mapping

$$
g_{a_{1} a_{2}}:\left|K_{a_{2}}^{(n+1)}\right| \rightarrow\left|\left(K_{a_{1}}^{k}\right)^{(n)}\right|, \quad k=k\left(a_{1}, a_{2}\right)
$$

by applying Lemma 5 to $K=K_{a_{2}}, L=K_{a_{1}}^{k}$, and $p=p_{a_{1} a_{2}}$. Lemma 5 is applicable because of (4) and Lemma 4 and yields the following conclusion.

Lemma 6. If $a_{1}<a_{2}$, then $g_{a_{1} a_{2}}$ satisfies

$$
d\left(g_{a_{1} a_{2}}, p_{a_{1} a_{2}}|| K_{a_{2}}^{(n+1)} \mid\right) \leq 2 \operatorname{mesh}\left(K_{a_{1}}^{k}\right), \quad k=k\left(a_{1}, a_{2}\right) .
$$

For $a \in A$ we now put

$$
Z_{a}^{*}=Z_{K_{a}}^{*}
$$

(see §5). For $a_{1}<^{\prime} a_{2}$ we define $r_{a_{1} a_{2}}: Z_{a_{2}}^{*} \rightarrow Z_{a_{1}}^{*}$ by

$$
r_{a_{1} a_{2}}=g_{a_{1} a_{2}} q_{0 a_{2}}^{*} \text {. }
$$

Here $q_{0 a_{2}}^{*}: Z_{a_{2}}^{*} \rightarrow\left|K_{a_{2}}^{(n)}\right|$ is the mapping $q_{0}^{*}: Z_{K}^{*} \rightarrow\left|K^{(n)}\right|$ from $\S 5$. Note that

$$
r_{a_{1} a_{2}}\left(Z_{a_{2}}^{*}\right) \subseteq\left|\left(K_{a_{1}}^{k}\right)^{(n)}\right|, \quad k=k\left(a_{1}, a_{2}\right)
$$

Lemma 7. $\mathbf{Z}=\left(Z_{a}^{*}, \varepsilon_{a}, r_{a a^{\prime}}, A^{\prime}\right)$ is an approximate system of nonempty metric compacta $Z_{a}^{*}$ with $\operatorname{dim} Z_{a}^{*} \leq n$. The limit $Z=\lim Z$ is a nonempty compact Hausdorff space with $\operatorname{dim} Z \leq n$ and $w(Z) \leq \operatorname{card}\left(A^{\prime}\right)=\operatorname{card}(A) \leq w(X)$. 
The proof of Lemma 7 is given in the next section.

\section{Verifying (A1)-(A3) FOR $Z$. The SPACE $Z$}

Proof of Lemma 7. For each $a \in A, \operatorname{dim} X_{a} \geq n$ and therefore $\left|\left(K_{a}^{k}\right)^{(n)}\right| \neq$ $\varnothing, k \geq 0$. It follows by $\S 5(4)$, that $Z_{a} \neq \varnothing$ and $Z_{a}^{*} \neq \varnothing$. Moreover, by $\S 5(15), \operatorname{dim} Z_{a}^{*} \leq n$.

We will now verify conditions $(\mathrm{A} 1)-(\mathrm{A} 3)$ for $\mathrm{Z}$. Let $a_{1}<^{\prime} a_{2}<^{\prime} a_{3}$. (A1) and (A2) require certain upper bounds for $d\left(r_{a_{1} a_{2}} r_{a_{2} a_{3}}, r_{a_{1} a_{3}}\right)$. By (19) of $\S 6$, it suffices to find the appropriate bounds for $d\left(r_{a_{1} a_{2}} g_{a_{2} a_{3}}|| K_{a_{3}}^{(n)}\left|, g_{a_{1} a_{3}}\right|\left|K_{a_{3}}^{(n)}\right|\right)$. By $\S 5(17)$ and $\S 5(1)$ we have

$$
\begin{gathered}
q_{0 a_{2}}^{*}||\left(K_{a_{2}}^{k}\right)^{(n)}\left|=q_{0 k a_{2}}\right|\left|\left(K_{a_{2}}^{k}\right)^{(n)}\right|, \\
d\left(q_{0 a_{2}}^{*}||\left(K_{a_{2}}^{k}\right)^{(n)}|, \quad 1|\left|\left(K_{a_{2}}^{k}\right)^{(n)}\right|\right) \leq \operatorname{mesh}\left(K_{a_{2}}\right),
\end{gathered}
$$

and therefore,

$$
d\left(q_{0 a_{2}}^{*} g_{a_{2} a_{3}}, g_{a_{2} a_{3}}\right) \leq \operatorname{mesh}\left(K_{a_{2}}\right) .
$$

On the other hand, $\S 6(17)$ yields

(4) $\quad d\left(g_{a_{2} a_{3}}, p_{a_{2} a_{3}}|| K_{a_{3}}^{(n+1)} \mid\right) \leq 2 \operatorname{mesh}\left(K_{a_{2}}^{k}\right) \leq 2 \operatorname{mesh}\left(K_{a_{2}}\right), \quad k=k\left(a_{2}, a_{3}\right)$.

Now (3), (4) and $\S 6(1)$ yield

$$
d\left(q_{0 a_{2}}^{*} g_{a_{2} a_{3}}, p_{a_{2} a_{3}}|| K_{a_{3}}^{(n+1)} \mid\right) \leq 3 \operatorname{mesh}\left(K_{a_{2}}\right) \leq \varepsilon_{a_{2}} .
$$

By $\S 6(3),(5)$ implies

(6) $\quad d\left(p_{a_{1} a_{2}} q_{0 a_{2}}^{*} g_{a_{2} a_{3}}, p_{a_{1} a_{2}} p_{a_{2} a_{3}}|| K_{a_{3}}^{(n+1)} \mid\right) \leq \operatorname{mesh}\left(K_{a_{1}}^{k\left(a_{1}, a_{2}\right)}\right) \leq \operatorname{mesh}\left(K_{a_{1}}\right)$.

Moreover, by $\S 6(17)$,

$$
d\left(g_{a_{1} a_{2}}, p_{a_{1} a_{2}}|| K_{a_{2}}^{(n+1)} \mid\right) \leq 2 \operatorname{mesh}\left(K_{a_{1}}^{k\left(a_{1}, a_{2}\right)}\right) \leq 2 \operatorname{mesh}\left(K_{a_{1}}\right),
$$

and therefore

(8) $\quad d\left(g_{a_{1} a_{2}} q_{0 a_{2}}^{*} g_{a_{2} a_{3}}, p_{a_{1} a_{2}} q_{0 a_{2}}^{*} g_{a_{2} a_{3}}\right) \leq 2 \operatorname{mesh}\left(K_{a_{1}}^{k\left(a_{1}, a_{2}\right)}\right) \leq 2 \operatorname{mesh}\left(K_{a_{1}}\right)$.

Now (6) and (8) yield

(9) $\quad d\left(g_{a_{1} a_{2}} q_{0 a_{2}}^{*} g_{a_{2} a_{3}}, p_{a_{1} a_{2}} p_{a_{2} a_{3}}|| K_{a_{3}}^{(n+1)} \mid\right) \leq 3 \operatorname{mesh}\left(K_{a_{1}}^{k\left(a_{1}, a_{2}\right)}\right) \leq 3 \operatorname{mesh}\left(K_{a_{1}}\right)$.

Furthermore, by $\S 6(2)$ we have

$$
d\left(p_{a_{1} a_{2}} p_{a_{2} a_{3}}, p_{a_{1} a_{3}}\right) \leq \operatorname{mesh}\left(K_{a_{1}}^{k\left(a_{1}, a_{2}\right)}\right) \leq \operatorname{mesh}\left(K_{a_{1}}\right),
$$

and by $\S 6(17)$ we have

$$
\begin{aligned}
d\left(g_{a_{1} a_{3}}, p_{a_{1} a_{3}}|| K_{a_{3}}^{(n+1)} \mid\right) & \leq 2 \operatorname{mesh}\left(K_{a_{1}}^{k\left(a_{1}, a_{3}\right)}\right) \leq 2 \operatorname{mesh}\left(K_{a_{1}}^{k\left(a_{1}, a_{2}\right)}\right) \\
& \leq 2 \operatorname{mesh} K_{a_{1}}
\end{aligned}
$$

(use $a_{2} \leq a_{3}$ and $\S 6(9)$ ). 
Finally, (9), (10), (11) and $\S 6(1)$ yield

$$
d\left(g_{a_{1} a_{2}} q_{0 a_{2}}^{*} g_{a_{2} a_{3}}, g_{a_{1} a_{3}}\right) \leq 6 \operatorname{mesh}\left(K_{a_{1}}^{k\left(a_{1}, a_{2}\right)}\right) \leq 6 \operatorname{mesh}\left(K_{a_{1}}\right) \leq \varepsilon_{a_{1}} .
$$

Since $r_{a_{1} a_{2}} r_{a_{2} a_{3}}=g_{a_{1} a_{2}} q_{0 a_{2}}^{*} g_{a_{2} a_{3}} q_{0 a_{3}}^{*}$ and $r_{a_{1} a_{3}}=g_{a_{1} a_{3}} q_{0 a_{3}}^{*}$, (12) yields

$$
d\left(r_{a_{1} a_{2}} r_{a_{2} a_{3}}, r_{a_{1} a_{3}}\right) \leq 6 \operatorname{mesh}\left(K_{a_{1}}^{k\left(a_{1}, a_{2}\right)}\right) \leq \varepsilon_{a_{1}},
$$

which verifies $(\mathrm{A} 1)$.

To obtain (A2), for given $a_{1}$ and $\eta>0$ choose an integer $k$ so large that

$$
6 \operatorname{mesh}\left(K_{a_{1}}^{k}\right) \leq \eta \text {. }
$$

By Lemma 2, there is an index $a_{2}>a_{1}$ such that $\S 6(2)-(4)$ hold for $k, a_{1}, a_{2}$. This proves that $a_{1}<^{\prime} a_{2}$. Since $k\left(a_{1}, a_{2}\right)$ is the maximal $k$ with these properties, we have $k \leq k\left(a_{1}, a_{2}\right)$ and therefore

$$
6 \operatorname{mesh}\left(K_{a_{1}}^{k\left(a_{1}, a_{2}\right)}\right) \leq \eta \text {. }
$$

Now let $a_{3}$ be any index with $a_{2}<^{\prime} a_{3}$. Then (13) and (15) imply

$$
d\left(r_{a_{1} a_{2}} r_{a_{2} a_{3}}, r_{a_{1} a_{3}}\right) \leq \eta
$$

which establishes (A2).

We now prove (A3). As above, for a given $a_{1} \in A$ and $\eta>0$ there is an $a_{2} \in A, a_{1}<a_{2}$, such that (15) holds. By (A3) for $\mathbf{X}$, we can assume that for any $a_{3}>a_{2}$ and any $y, y^{\prime} \in\left|K_{a_{3}}^{(n)}\right|$,

$$
d\left(y, y^{\prime}\right) \leq \varepsilon_{a_{3}} \Rightarrow d\left(p_{a_{1} a_{3}}(y), p_{a_{1} a_{3}}\left(y^{\prime}\right)\right) \leq \operatorname{mesh}\left(K_{a_{1}}^{k\left(a_{1}, a_{2}\right)}\right) .
$$

(17) and (11) (applied to $y$ and $y^{\prime}$ ) and (15) yield

$$
\begin{aligned}
d\left(y, y^{\prime}\right) \leq \varepsilon_{a_{3}} \Rightarrow d\left(g_{a_{1} a_{3}}(y), g_{a_{1} a_{3}}\left(y^{\prime}\right)\right) \leq 5 \operatorname{mesh}\left(K_{a_{1}}^{k\left(a_{1}, a_{2}\right)}\right) \leq \eta \\
y, y^{\prime} \in\left|K_{a_{3}}^{(n)}\right| .
\end{aligned}
$$

Now let $x, x^{\prime} \in Z_{a_{3}}^{*}$. Then $y=q_{0 a_{3}}^{*}(x), y^{\prime}=q_{0 a_{3}}^{*}\left(x^{\prime}\right) \in\left|K_{a_{3}}^{(n)}\right|$. Moreover, by $\S 5(27)$,

$$
d\left(y, y^{\prime}\right) \leq d^{*}\left(x, x^{\prime}\right)
$$

Therefore, $d^{*}\left(x, x^{\prime}\right) \leq \varepsilon_{a_{3}}$ implies $d\left(y, y^{\prime}\right) \leq \varepsilon_{a_{3}}$, and (18) yields

$$
d\left(r_{a_{1} a_{3}}(x), r_{a_{1} a_{3}}\left(x^{\prime}\right)\right) \leq 5 \operatorname{mesh}\left(K_{a_{1}}^{k\left(a_{1}, a_{2}\right)}\right) \leq \eta .
$$

To complete the proof of Lemma 7, note that Propositions 1 and 5 imply that $Z=\lim Z$ is a nonempty compact Hausdorff space with $\operatorname{dim} Z \leq n$. Moreover, by Propositions 2 and 12, $w(Z) \leq \operatorname{card}\left(A^{\prime}\right)=\operatorname{card}(A) \leq w(X)$. We will denote the natural projections from $Z$ to $Z_{a}^{*}, a \in A$, by $r_{a}: Z \rightarrow Z_{a}^{*}$. 
Remark 4. For given $a \in A$ and integer $k \geq 0$ consider the sets

$$
C_{a}^{k}=\left|K_{a}^{(n)}\right| \oplus \cdots \oplus\left|\left(K_{a}^{k}\right)^{(n)}\right|, \quad D_{a}^{k}=Z_{a} \oplus\left|\left(K_{a}^{k+1}\right)^{(n)}\right| \oplus \cdots .
$$

They are disjoint and are closed in $Z_{a}^{*}, Z_{a}^{*}=C_{a}^{k} \cup D_{a}^{k}$. Let $\eta>0$ be such that $d\left(C_{a}^{k}, D_{a}^{k}\right)>\eta$. For sufficiently large $a^{\prime \prime}$ one has $d\left(r_{a a^{\prime \prime}} r_{a^{\prime \prime}}, r_{a}\right) \leq \eta$ and $k\left(a, a^{\prime \prime}\right) \geq k+1$. Therefore, $r_{a a^{\prime \prime}} r_{a^{\prime \prime}}\left(Z_{a}^{*}\right) \subseteq\left|\left(K_{a}^{k\left(a, a^{\prime \prime}\right)}\right)^{(n)}\right| \subseteq D_{a}^{k}$ and thus also $r_{a}\left(Z_{a}^{*}\right) \subseteq D_{a}^{k}$. This implies that $r_{a}\left(Z_{a}^{*}\right) \cap\left|\left(K_{a}^{k}\right)^{(n)}\right|=\varnothing$ for all $k$ and therefore, $r_{a}\left(Z_{a}^{*}\right) \subseteq Z_{a}$. Nevertheless, $Z_{a} \cap r_{a a^{\prime}}\left(Z_{a^{\prime}}\right) \subseteq Z_{a} \cap r_{a a^{\prime}}\left(Z_{a^{\prime}}^{*}\right)=\varnothing$ for every $a<a^{\prime}$.

\section{The MAPPING $f: Z \rightarrow X$}

In order to define the mapping $f$ we first establish a lemma about maps $f_{a}^{*}: Z_{a}^{*} \rightarrow X_{a}$, defined by $f_{a}^{*}=f_{K_{a}}^{*}($ see $\S 5(19))$.

Lemma 8. If $a_{1}<a_{2}$, then

$$
d\left(f_{a_{1}}^{*} r_{a_{1} a_{2}}, p_{a_{1} a_{2}} f_{a_{2}}^{*}\right) \leq 3 \operatorname{mesh}\left(K_{a_{1}}^{k\left(a_{1}, a_{2}\right)}\right) \leq \varepsilon_{a_{1}} .
$$

Proof. By $\S 5(24)$ (for $k=0$ ), we have

$$
d\left(f_{a_{2}}^{*}, q_{0 a_{2}}^{*}\right) \leq \operatorname{mesh}\left(K_{a_{2}}\right) \leq \varepsilon_{a_{2}}
$$

and therefore, by $\S 6(3)$,

$$
d\left(p_{a_{1} a_{2}} f_{a_{2}}^{*}, p_{a_{1} a_{2}} q_{0 a_{2}}^{*}\right) \leq \operatorname{mesh}\left(K_{a_{1}}^{k\left(a_{1}, a_{2}\right)}\right) .
$$

Using $§ 6(17)$, we also have

$$
d\left(g_{a_{1} a_{2}} q_{0 a_{2}}^{*}, p_{a_{1} a_{2}} q_{0 a_{2}}^{*}\right) \leq 2 \operatorname{mesh}\left(K_{a_{1}}^{k\left(a_{1}, a_{2}\right)}\right) .
$$

Since $r_{a_{1} a_{2}}=g_{a_{1} a_{2}} q_{0 a_{2}}^{*},(3)$ and (4) yield

$$
d\left(r_{a_{1} a_{2}}, p_{a_{1} a_{2}} f_{a_{2}}^{*}\right) \leq 3 \operatorname{mesh}\left(K_{a_{1}}^{k\left(a_{1}, a_{2}\right)}\right) \leq \varepsilon_{a_{1}} .
$$

Since $r_{a_{1} a_{2}}: Z_{a_{2}}^{*} \rightarrow\left|\left(K_{a_{1}}^{k\left(a_{1}, a_{2}\right)}\right)^{(n)}\right| \subseteq X_{a_{1}}$ and $f_{a_{1}}^{*}||\left(K_{a_{1}}^{k}\right)^{(n)} \mid$ is the inclusion $\left|\left(K_{a_{1}}^{k}\right)^{(n)}\right| \rightarrow X_{a_{1}}$ (see $\left.\S 5(21)\right)$, (5) is the desired formula (1).

Lemma 9. For any $a_{1} \in A$ and $\eta>0$, there is an $a_{2} \in A, a_{1}<a_{2}$, such that for any $a^{\prime \prime} \geq a_{2}$, one has

$$
d\left(f_{a_{1}}^{*} r_{a_{1} a^{\prime \prime}}, p_{a_{1} a^{\prime \prime}} f_{a^{\prime \prime}}^{*}\right) \leq \eta
$$

Proof. Choose an integer $k \geq 0$ such that $\S 7(14)$ holds. Repeating the argument which led to $\S 7(15)$, we find an $a_{2} \in A, a_{1}<a_{2}$, such that $\S 7(15)$ holds. Applying Lemma 8 to any $a^{\prime \prime} \geq a_{2}$ we obtain

$$
d\left(f_{a_{1}}^{*} r_{a_{1} a^{\prime \prime}}, p_{a_{1} a^{\prime \prime}} f_{a^{\prime \prime}}^{*}\right) \leq \eta, \quad a^{\prime \prime} \geq a_{2} .
$$

Lemma 10. There is a mapping $f: Z \rightarrow X$ such that

$$
f_{a}^{*} r_{a}=p_{a} f, \quad a \in A,
$$

where $r_{a}: Z \rightarrow Z_{a}^{*}$ are the natural projections. 
Proof. For a given $\eta>0$, choose $\delta>0$ so small that

$$
d\left(z, z^{\prime}\right) \leq \delta \Rightarrow d\left(f_{a}^{*}(z), f_{a}^{*}\left(z^{\prime}\right)\right) \leq \eta .
$$

By Proposition 3 for $\mathbf{Z}^{*}$, there is an $a^{\prime} \in A, a<^{\prime} a^{\prime}$, such that for any $a^{\prime \prime} \geq a^{\prime}$

$$
d\left(r_{a a^{\prime \prime}} r_{a^{\prime \prime}}, r_{a}\right) \leq \delta
$$

and therefore,

$$
d\left(f_{a}^{*} r_{a a^{\prime \prime}} r_{a^{\prime \prime}}, f_{a}^{*} r_{a}\right) \leq \eta .
$$

On the other hand, by (6), for sufficiently large $a^{\prime \prime}$,

$$
d\left(f_{a}^{*} r_{a a^{\prime \prime}} r_{a^{\prime \prime}}, p_{a a^{\prime \prime}} f_{a^{\prime \prime}}^{*} r_{a^{\prime \prime}}\right) \leq \eta \text {. }
$$

(11) and (12) yield

$$
d\left(f_{a}^{*} r_{a}, p_{a a^{\prime \prime}} f_{a^{\prime \prime}}^{*} r_{a^{\prime \prime}}\right) \leq 2 \eta,
$$

for $a^{\prime \prime}$ sufficiently large. We have thus proved,

$$
\lim _{a^{\prime \prime}} p_{a a^{\prime \prime}} f_{a^{\prime \prime}}^{*} r_{a^{\prime \prime}}=f_{a}^{*} r_{a} \text {. }
$$

Let $f: Z \rightarrow \prod_{a \in A} X_{a}$ be the mapping given by (8). Formula (14) and Proposition 3 show that $f(Z) \subseteq X=\lim X$, so that one can view $f$ as a mapping $f: Z \rightarrow X$.

Remark 5. Lemma 10 also follows from more general results (see [10, Remark 4]).

\section{Fibers of the MAPPING $f: Z \rightarrow X$}

For a given $x \in X$ we will now express the fiber $f^{-1}(x)$ in terms of the system $\mathbf{Z}$. We put

(1) $x_{a}=p_{a}(x), a \in A$,

(2) $N_{a}(x)=\left\{x^{\prime} \in X_{a}: d\left(x^{\prime}, x_{a}\right) \leq \varepsilon_{a}\right\}$.

We often abbreviate $N_{a}(x)$ to $N_{a}$.

Lemma 11. If $a_{1}<a_{2}$, then

(3) $x^{\prime} \in N_{a_{2}} \Rightarrow d\left(p_{a_{1} a_{2}}\left(x^{\prime}\right), x_{a_{1}}\right) \leq 2 \operatorname{mesh}\left(K_{a_{1}}^{k\left(a_{1}, a_{2}\right)}\right) \leq 2 \operatorname{mesh}\left(K_{a_{1}}\right) \leq \varepsilon_{a_{1}}$.

(4) $p_{a_{1} a_{2}}\left(N_{a_{2}}\right) \subseteq N_{a_{1}}$.

Proof. $x^{\prime} \in N_{a_{2}}$ implies $d\left(x^{\prime}, x_{a_{2}}\right) \leq \varepsilon_{a_{2}}$, and therefore by $\S 6(3)$,

(5) $d\left(p_{a_{1} a_{2}}\left(x^{\prime}\right), p_{a_{1} a_{2}}\left(x_{a_{2}}\right)\right) \leq \operatorname{mesh}\left(K_{a_{1}}^{k\left(a_{1}, a_{2}\right)}\right)$.

Moreover, by (1) and $\S 6(8)$,

(6) $d\left(p_{a_{1} a_{2}}\left(x_{a_{2}}\right), x_{a_{1}}\right) \leq \operatorname{mesh}\left(K_{a_{1}}^{k\left(a_{1}, a_{2}\right)}\right)$.

Now, (5) and (6) yield (3). Formula (4) is an immediate consequence of (3).

It is a consequence of Lemma 11 that

$$
\mathbf{N}(x)=\left(N_{a}(x), \varepsilon_{a}, p_{a a^{\prime}}, A^{\prime}\right)
$$

is an approximate system. 
Lemma 12. The limit of the approximate system $\mathbf{N}(x)$ is $\{x\}$.

Proof. Since $p_{a}(x)=x_{a} \in N_{a}$, the point $x$ belongs to $\lim \mathbf{N}(x)$. Now assume that $x^{\prime} \in X$ belongs to $\lim \mathbf{N}(x)$. We will show that $p_{a}\left(x^{\prime}\right)=x_{a}$ for every $a \in A$ and therefore $x^{\prime}=x$.

By (A3) for $\mathbf{X}$, there is an $a^{\prime}, a<^{\prime} a^{\prime}$, such that for every $a^{\prime \prime} \geq a^{\prime}$,

$$
d\left(y, y^{\prime}\right) \leq \varepsilon_{a^{\prime \prime}} \Rightarrow d\left(p_{a a^{\prime \prime}}(y), p_{a a^{\prime \prime}}\left(y^{\prime}\right)\right) \leq \eta .
$$

Since $p_{a^{\prime \prime}}\left(x^{\prime}\right) \in N_{a^{\prime \prime}}(x)$, we have $d\left(p_{a^{\prime \prime}}\left(x^{\prime}\right), x_{a^{\prime \prime}}\right) \leq \varepsilon_{a^{\prime \prime}}$, and therefore,

$$
d\left(p_{a a^{\prime \prime}} p_{a^{\prime \prime}}\left(x^{\prime}\right), p_{a a^{\prime \prime}}\left(x_{a^{\prime \prime}}\right)\right) \leq \eta \text {. }
$$

Passing to the limit with $a^{\prime \prime}$ we obtain (using Proposition 3 )

$$
d\left(p_{a}\left(x^{\prime}\right), x_{a}\right) \leq \eta \text {. }
$$

Since $\eta>0$ was arbitrary, we conclude that indeed $p_{a}\left(x^{\prime}\right)=x_{a}$ for all $a \in A$.

For $a \in A$ and $x \in X$ put

$$
M_{a}=M_{a}(x)=f_{a}^{*-1}\left(N_{a}\right) .
$$

Lemma 13. If $a_{1}<^{\prime} a_{2}$, then

$$
r_{a_{1} a_{2}}\left(M_{a_{2}}\right) \subseteq M_{a_{1}} .
$$

Proof. Let $y \in M_{a_{2}}=f_{a_{2}}^{*-1}\left(N_{a}\right)$. Then $x^{\prime}=f_{a_{2}}^{*}(y) \in N_{a_{2}}$. By (3) we obtain

$$
d\left(p_{a_{1} a_{2}}\left(x^{\prime}\right), x_{a_{1}}\right) \leq 2 \operatorname{mesh}\left(K_{a_{1}}\right) .
$$

On the other hand, by $\S 8(1)$,

$$
d\left(f_{a_{1}}^{*} r_{a_{1} a_{2}}(y), p_{a_{1} a_{2}} f_{a_{2}}^{*}(y)\right) \leq 3 \operatorname{mesh}\left(K_{a_{1}}\right),
$$

so that we obtain

$$
d\left(f_{a_{1}}^{*} r_{a_{1} a_{2}}(y), x_{a_{1}}\right) \leq 5 \operatorname{mesh}\left(K_{a_{1}}\right) \leq \varepsilon_{a_{1}} .
$$

This proves that $f_{a_{1}}^{*} r_{a_{1} a_{2}}(y) \in N_{a_{1}}$, i.e., $r_{a_{1} a_{2}}(y) \in M_{a_{1}}$.

It is a consequence of Lemma 13 that

$$
\mathbf{M}(x)=\left(M_{a}(x), \varepsilon_{a}, r_{a a^{\prime}}, A^{\prime}\right)
$$

is an approximate system.

Lemma 14. The limit of the approximate system $\mathbf{M}(x)$ is $f^{-1}(x)$.

Proof. Let $z \in f^{-1}(x)$. By $\S 8(8)$, we have

$$
f_{a}^{*} r_{a}(z)=p_{a} f(z)=p_{a}(x)=x_{a} \in N_{a}(x)
$$

so that $r_{a}(z) \in f_{a}^{*-1}\left(N_{a}(x)\right)=M_{a}(x)$. This shows that

$$
f^{-1}(x) \subseteq \lim \mathbf{M}(x) .
$$


Conversely, assume that $z \in Z$ belongs to $\lim \mathbf{M}(x)$. Then $r_{a}(z) \in M_{a}=$ $f_{a}^{*-1}\left(N_{a}(x)\right)$ and therefore $f_{a}^{*} r_{a}(z) \in N_{a}(x)$. By $\S 8(8), p_{a} f(z)=f_{a}^{*} r_{a}(z) \in$ $N_{a}(x)$, so that $f(z)$ belongs to $\lim \mathbf{N}(x)=\{x\}$ (Lemma 12). Consequently, $f(z)=x$, i.e., $z \in f^{-1}(x)$.

Lemma 15. The mapping $f: Z \rightarrow X$ is onto.

Proof. Let $x \in X$. We will first show that

$$
M_{a}(x) \neq \varnothing, \quad a \in A .
$$

We have shown in $\S 5$ that $f_{K}: Z_{K} \rightarrow|K|$ is an onto mapping. Since $Z_{K} \subseteq Z_{K}^{*}$ and $f_{K}^{*} \mid Z_{K}=f_{K}$, we see that also $f_{K}^{*}: Z_{K}^{*} \rightarrow|K|$ is onto. This means that all the maps $f_{a}^{*}: Z_{a}^{*} \rightarrow X_{a}$ are onto. For any $x \in X, N_{a}(x) \neq \varnothing$ because it contains $x_{a}=p_{a}(x)$. Therefore, also $M_{a}(x)=f_{a}^{*-1}\left(N_{a}(x)\right) \neq \varnothing$.

We now use Lemma 14 and Proposition 1 to conclude that $f^{-1}(x) \neq \varnothing$.

$$
\text { 10. } f: Z \rightarrow X \text { IS CELL-LIKE. }
$$

We first establish an important technical lemma.

Lemma 16. Let $a_{1}<a_{2}$ and $j \geq 0$ be any integer. Then the restriction of $r_{a_{1} a_{2}}$ to $\left|\left(K_{a_{2}}^{j}\right)^{(n)}\right|$ admits an extension

$$
\bar{r}_{a_{1} a_{2}}:\left|\left(K_{a_{2}}^{j}\right)^{(n+1)}\right| \rightarrow\left|\left(K_{a_{1}}^{k}\right)^{(n)}\right|, \quad k=k\left(a_{1}, a_{2}\right)
$$

Moreover,

$$
d\left(\bar{r}_{a_{1} a_{2}}, p_{a_{1} a_{2}}||\left(K_{a_{2}}^{j}\right)^{(n+1)} \mid\right) \leq 3 \operatorname{mesh}\left(K_{a_{1}}\right) .
$$

Proof. Recall that $r_{a_{1} a_{2}}=g_{a_{1} a_{2}} q_{0 a_{2}}^{*}(\S 6(19))$. Therefore, the considered restriction equals $g_{a_{1} a_{2}} q_{0 j a_{2}}(\S 5(17))$. However, $q_{0 j a_{2}}: K_{a_{2}}^{j} \rightarrow K_{a_{2}}$ is a simplicial approximation of the identity (§5). Therefore, $q_{0 a_{2}}^{*}$ maps $\left|\left(K_{a_{2}}^{j}\right)^{(n+1)}\right|$ into $\left|K_{a_{2}}^{(n+1)}\right|$ and for any $z \in\left|\left(K_{a_{2}}^{j}\right)^{(n+1)}\right|$, one has

$$
d\left(q_{0 a_{2}}^{*}(z), z\right) \leq \operatorname{mesh} K_{a_{2}} \leq \varepsilon_{a_{2}} .
$$

Since $g_{a_{1} a_{2}}$ was actually defined on $\left|\left(K_{a_{2}}\right)^{(n+1)}\right|(\S 6(16))$, we see that $r_{a_{1} a_{2}}$ has an extension $\bar{r}_{a_{1} a_{2}}$ as required by (1).

For any $z \in\left|K_{a_{2}}^{j}\right|,(3)$ and $\S 6(3)$ yield

$$
d\left(p_{a_{1} a_{2}} q_{0 a_{2}}^{*}(z), p_{a_{1} a_{2}}(z)\right) \leq \operatorname{mesh}\left(K_{a_{1}}\right) .
$$

On the other hand, by $\S 6(17)$, we have

$$
d\left(p_{a_{1} a_{2}} q_{0 a_{2}}^{*}(z), g_{a_{1} a_{2}} q_{0 a_{2}}^{*}(z)\right) \leq 2 \operatorname{mesh}\left(K_{a_{1}}^{k}\right) .
$$

Now (4) and (5) yield (2). 
Lemma 17. For every $x \in X$, the fiber $f^{-1}(x)$ has trivial shape, and therefore $f: Z \rightarrow X$ is a cell-like mapping.

Proof. Let $a_{1} \in A$ be arbitrary. Choose any $a_{2} \in A, a_{1}<^{\prime} a_{2}$. In view of Lemma 14 and Proposition 8, it suffices to exhibit an $a_{2}^{\prime}, a_{2}<^{\prime} a_{2}^{\prime}$ such that for any $a_{3} \geq a_{2}^{\prime}$, there is a homotopy $G: M_{a_{3}}(x) \times I \rightarrow M_{a_{1}}(x)$ such that $G_{1}$ is constant and

$$
G_{0}=r_{a_{1} a_{2}} r_{a_{2} a_{3}} \mid M_{a_{3}}(x) \text {. }
$$

Note that $N_{a_{2}}(x)$ is a neighborhood of $x_{a_{2}}=p_{a_{2}}(x)$ in $X_{a_{2}}$ (see §9). Since $X_{a_{2}}$ is a polyhedron, there is a polyhedral neighborhood $U^{a_{2}}$ of $x_{a_{2}}$ which is contained in $N_{a_{2}}(x)$ and is contractible in itself. One can achieve that $U=|L|$ where $L$ is a subcomplex of the $j$ th barycentric subdivision $K_{a_{2}}^{j}$ of $K_{a_{2}}$ for some sufficiently large $j$.

Choose $\eta>0$ so small that

$$
d\left(x^{\prime}, x_{a_{2}}\right) \leq 3 \eta \Rightarrow x^{\prime} \in|L|
$$

and choose $k \geq j$ so large that

$$
3 \text { mesh } K_{a_{2}}^{j} \leq \eta
$$

By Lemma 2, there is an $a_{2}^{\prime}, a_{2}<a_{2}^{\prime}$, such that $k\left(a_{2}, a_{2}^{\prime}\right) \geq k$. Therefore, by Lemma 8 , for any $a_{3}, a_{2}^{\prime}<a_{3}$, one has

$$
d\left(f_{a_{2}}^{*} r_{a_{2} a_{3}}, p_{a_{2} a_{3}} f_{a_{3}}^{*}\right) \leq \eta .
$$

By Proposition 3 and (A3), one can also assume that for $a_{3}>a_{2}^{\prime}$

$$
\begin{gathered}
d\left(p_{a_{2} a_{3}}\left(x_{a_{3}}\right), x_{a_{2}}\right) \leq \eta, \\
d\left(y, y^{\prime}\right) \leq \varepsilon_{a_{3}} \Rightarrow d\left(p_{a_{2} a_{3}}(y), p_{a_{2} a_{3}}\left(y^{\prime}\right)\right) \leq \eta .
\end{gathered}
$$

We will now show that

$$
r_{a_{2} a_{3}}\left(M_{a_{3}}(x)\right) \subseteq|L| \text {. }
$$

Indeed, if $z \in M_{a_{3}}(x)=f_{a_{3}}^{*-1}\left(N_{a_{3}}(x)\right)$ (see $\left.\S 9(11)\right)$, then $f_{a_{3}}^{*}(z) \in N_{a_{3}}(x)$, and therefore,

$$
d\left(f_{a_{3}}^{*}(z), x_{a_{3}}\right) \leq \varepsilon_{a_{3}}
$$

This and (11) yield

$$
d\left(p_{a_{2} a_{3}} f_{a_{3}}^{*}(z), p_{a_{2} a_{3}}\left(x_{a_{3}}\right)\right) \leq \eta .
$$

Now (9), (10) and (14) yield

$$
d\left(f_{a_{2}}^{*} r_{a_{2} a_{3}}(z), x_{a_{2}}\right) \leq 3 \eta .
$$

Therefore (7) implies

$$
f_{a_{2}}^{*} r_{a_{2} a_{3}}(z) \in|L| \text {. }
$$


This establishes (12) because

$$
r_{a_{2} a_{3}}\left(Z_{a_{3}}^{*}\right) \subseteq\left|\left(K_{a_{2}}^{k\left(a_{2}, a_{3}\right)}\right)^{(n)}\right|
$$

and the restriction of $f_{a_{2}}^{*}$ to the right side of (17) is an inclusion $(\S 5(21))$.

Since $L$ is a subcomplex of $K_{a_{2}}^{j}$ and $k\left(a_{2}, a_{3}\right) \geq k\left(a_{2}, a_{2}^{\prime}\right) \geq k \geq j$, there is an $i$ such that the $i$ th barycentric subdivision $L^{i}$ of $L$ is a subcomplex of $K_{a_{2}}^{k\left(a_{2}, a_{3}\right)}$. Therefore for any integer $m \geq 0$

$$
\left|L^{i}\right| \cap\left|\left(K_{a_{2}}^{k\left(a_{2}, a_{3}\right)}\right)^{(m)}\right|=\left|\left(L^{i}\right)^{(m)}\right| .
$$

Since $|L|=\left|L^{i}\right|$ is contractible in itself, there is a homotopy $H:\left|L^{i}\right| \times I \rightarrow$ $\left|L^{i}\right|$ such that

$$
\begin{aligned}
& H_{0}=\mathrm{id}, \\
& H_{1}=v,
\end{aligned}
$$

where $v$ is a vertex of $L^{i}$. We now restrict $H$ to $\left|\left(L^{i}\right)^{(n)}\right| \times I$. Note that this is a polyhedron of dimension $\leq n+1$ and $H||\left(L^{i}\right)^{(n)} \mid \times \partial I$ is a PL-mapping. By the relative simplicial approximation theorem (see [15, Chapter $3, \S 4.1]$ ), we can assume that $H||\left(L^{i}\right)^{(n)}|\times I \rightarrow| L^{i} \mid$ is a PL-mapping. Then

$$
\operatorname{dim} H\left(\left|\left(L^{i}\right)^{(n)}\right| \times I\right) \leq n+1 .
$$

It is now possible to "push" $H$ off the simplexes of $L^{i}$ of dimensions $>n+1$. One can therefore assume that $H$ also satisfies

$$
H\left(\left|\left(L^{i}\right)^{(n)}\right| \times I\right) \subseteq\left|\left(L^{i}\right)^{(n+1)}\right| .
$$

If $z \in M_{a_{3}}(x)$, then by (12), (17) and (18) (for $m=n$ ),

$$
r_{a_{2} a_{3}}(z) \in\left|\left(L^{i}\right)^{(n)}\right| .
$$

Therefore, for $(z, t) \in M_{a_{3}}(x) \times I$, by (22) and (18) (for $\left.m=n+1\right)$, we have

$$
x^{\prime}=H\left(r_{a_{2} a_{3}}(z), t\right) \in\left|\left(L^{i}\right)^{(n+1)}\right| \subseteq\left|\left(K_{a_{2}}^{k\left(a_{2}, a_{3}\right)}\right)^{(n+1)}\right| .
$$

However, Lemma 16 yields a mapping

$$
\bar{r}_{a_{1} a_{2}}:\left|\left(K_{a_{2}}^{k\left(a_{2}, a_{3}\right)}\right)^{(n+1)}\right| \rightarrow\left|\left(K_{a_{1}}^{k\left(a_{1}, a_{2}\right)}\right)^{(n)}\right| \subseteq Z_{a_{1}}^{*} .
$$

We conclude that

$$
G=\bar{r}_{a_{1} a_{2}} H\left(r_{a_{2} a_{3}} \times 1\right): M_{a_{3}}(x) \times I \rightarrow Z_{a_{1}}^{*}
$$

is a well-defined mapping.

By (24), $x^{\prime} \in|L| \subseteq N_{a_{2}}(x)$, and therefore by $\S 9(3)$,

$$
d\left(p_{a_{1} a_{2}}\left(x^{\prime}\right), x_{a_{1}}\right) \leq 2 \operatorname{mesh}\left(K_{a_{1}}\right) .
$$


By (25) and (2) we also have

$$
d\left(\bar{r}_{a_{1} a_{2}}\left(x^{\prime}\right), p_{a_{1} a_{2}}\left(x^{\prime}\right)\right) \leq 3 \operatorname{mesh}\left(K_{a_{1}}\right),
$$

so that

$$
d\left(G(z, t), x_{a_{1}}\right) \leq 5 \operatorname{mesh}\left(K_{a_{1}}\right) \leq \varepsilon_{a_{1}} .
$$

This proves that $f_{a_{1}}^{*} G(z, t)=G(z, t) \in N_{a_{1}}(x)$. Therefore,

$$
G\left(M_{a_{3}}(x) \times I\right) \subseteq M_{a_{1}}(x),
$$

and $G$ is a homotopy $G: M_{a_{3}}(x) \times I \rightarrow M_{a_{1}}(x)$.

The map $G_{1}$ is constant because $H_{1}$ is constant. $G_{0}=r_{a_{1} a_{2}} r_{a_{2} a_{3}}$ follows from (19), (24) and the fact that $\bar{r}_{a_{1} a_{2}}$ extends $r_{a_{1} a_{2}} \mid\left(K_{a_{2}}^{k\left(a_{2}, a_{3}\right)}\right)^{(n)}$ (see Lemma 16).

\section{Cell-Like images of $n$-Dimensional compact SPaCes.}

We now state our main result.

Theorem 3. Let $X$ be a compact Hausdorff space whose cohomological dimension $\operatorname{dim}_{\mathrm{Z}} X \leq n, n \geq 1$. Then there exist a compact Hausdorff space $Z$ of covering dimension $\operatorname{dim} Z \leq n$ and weight $w(Z) \leq w(X)$ and a cell-like mapping $f: Z \rightarrow X$.

Proof. If $\operatorname{dim}_{\mathrm{Z}} X=n, n \geq 1$, we apply Proposition 12 and obtain an approximate system $\mathbf{X}$. Using $\mathbf{X}$, we construct the approximate system $\mathbf{Z}$ of $\S 7$. By Lemma 7, $Z=\lim Z$ is a compact Hausdorff space with $\operatorname{dim} Z \leq n$ and $w(Z) \leq w(X)$. We define $f: Z \rightarrow X$ as in $\S 8$. By Lemma 17, $f$ is a cell-like mapping.

Corollary 1. Every compact metric space $X$ with $\operatorname{dim}_{\mathrm{z}} X \leq n, n \geq 1$, is the image of a metric compactum $Z, \operatorname{dim} Z \leq n$, under a cell-like mapping $f: Z \rightarrow$ $X$.

The next result gives a converse to Theorem 3 .

Proposition 13. If a paracompact space $X$ is the cell-like image of a normal space $Z$ with $\operatorname{dim} Z \leq n$, then $\operatorname{dim}_{\mathrm{Z}} X \leq n$.

Proof. Let $f: Z \rightarrow X$ be a cell-like mapping. Since $f$ is proper and $X$ is paracompact, one concludes that $Z$ also is paracompact (see [13, Chapter 2, Proposition 5.9]). By the standard definition of cohomological dimension (see [4]), we must show that for any closed subset $A \subseteq X$ the Čech cohomology $\check{H}^{m}(X, A ; \mathbf{Z})=0$, for $m \geq n+1$. Since $\operatorname{dim} Z \leq n$, we have $\check{H}^{m}(Z, B ; \mathbf{Z})=$ 0 , for any closed subset $B \subseteq Z$ and $m \geq n+1$. In particular, this holds for $B=f^{-1}(A)$. Therefore, it suffices to conclude that for all $m, f$ induces an isomorphism

$$
f^{*}: \check{H}^{m}(X, A ; \mathbf{Z}) \rightarrow \check{H}^{m}\left(Z, f^{-1}(A) ; \mathbf{Z}\right)
$$


Since the fibers $f^{-1}(x)$ are of trivial shape, their cohomology vanishes. Therefore the Vietoris-Begle theorem applies (see [15, Chapter $6, \S 9$, Theorem 15]) and yields the desired conclusion that $f^{*}$ is an isomorphism.

Remark 6. We do not know whether paracompact spaces $X$ with $\operatorname{dim}_{\mathrm{Z}} X \leq n$ are cell-like images of paracompact spaces $Z$ with $\operatorname{dim} Z \leq n$.

\section{REFERENCES}

1. A. N. Dranishnikov, On P. S. Aleksandrov's problem, Mat. Sb. 135 (4) (1988), 551-557. (Russian)

2. R. D. Edwards. A theorem and a question related to cohomological dimension and cell-like maps, Notices Amer. Math. Soc. 25 (1978), A-259.

3. Y. Kodama, Note on cohomological dimension for noncompact spaces, J. Math. Soc. Japan 18 (1966), 343-359.

4. V. I. Kuz' minov, Homological dimension theory, Uspehi Mat. Nauk 23 (1968), no. 5(143), 3-49=Russian Math. Surveys 23 (1968), no. 5, 1-45.

5. S. Mardešić, Factorization theorems for cohomological dimension, Topology Appl. 30 (1988), 291-306.

6. S. Mardešić and L. R. Rubin, Approximate inverse systems of compacta and covering dimension, Pacific J. Math. (to appear).

7. S. Mardešić and J. Segal, Shape theory, North-Holland, Amsterdam, 1982.

8. __, $\mathscr{P}$-like continua and approximate inverse limits, Math. Japon. (to appear).

9. __ Stability of almost commutative inverse systems of compacta, Topology Appl. (to appear).

10. _ Mapping approximate inverse systems of compacta, Fund. Math. (to appear).

11. B. A. Pasynkov, On the absence of polyhedral spectra of bicompacta, Dokl. Akad. Nauk SSSR 142 (1962), 546-549. (Russian)

12. __ On spectra and dimension of topological spaces, Mat. Sb. 57 (1962), 449-476. (Russian)

13. A. R. Pears, Dimension theory of general spaces, Cambridge Univ. Press, Cambridge, 1975.

14. L. R. Rubin and P. J. Schapiro, Cell-like maps onto noncompact spaces of finite cohomological dimension, Topology Appl. 27 (1987), 221-244.

15. E. Spanier, Algebraic topology, McGraw-Hill, New York, 1966.

16. J. J. Walsh, Dimension, cohomological dimension, and cell-like mappings, Shape Theory and Geometric Topology, Proc. Dubrovnik 1981, Lecture Notes in Math., vol. 870, SpringerVerlag, Berlin, 1981, pp. 105-118.

17. T. Watanabe, Approximate shape, I, Tsukuba J. Math. 11 (1987), 17-59.

Department of Mathematics, University of Zagreb, 41001 Zagreb, P. O. BoX 187, YUGLOSAVIA

Department of Mathematics, University of Oklahoma, 601 Elm Avenue, Room 423, NoRman, OKLAHOMA 73019 